\title{
Mathematical Models of Avascular Tumor Growth*
}

\section{Tiina Roose ${ }^{\dagger}$ \\ S. Jonathan Chapman Philip K. Maini ${ }^{\S}$}

\begin{abstract}
This review will outline a number of illustrative mathematical models describing the growth of avascular tumors. The aim of the review is to provide a relatively comprehensive list of existing models in this area and discuss several representative models in greater detail. In the latter part of the review, some possible future avenues of mathematical modeling of avascular tumor development are outlined together with a list of key questions.
\end{abstract}

Key words. tumor modeling, cancer modeling, avascular tumors, reaction-diffusion equation, soft tissue mechanics, cellular automata

AMS subject classifications. 93A $30,92 \mathrm{~B} 05,62 \mathrm{P} 10$

DOI. $10.1137 / \mathrm{S} 0036144504446291$

I. Introduction. Cancer is one of the main causes of morbidity and mortality in the world (http://www.who.org/). In the United Kingdom, one in four people will die of cancer, whilst one in three will at some point in their life be diagnosed to have cancer (http://www.cancerresearchuk.com/). Therefore, developed countries are investing large sums of money into cancer research in order to find cures and improve existing treatments. In comparison to molecular biology, cell biology, and drug delivery research, mathematics has so far contributed relatively little to the area. A search in the PubMed bibliographic database (http://www.ncbi.nlm.nih.gov/PubMed/) shows that out of 1.5 million papers in the area of cancer research, approximately $5 \%$ are concerned with mathematical modeling. ${ }^{1}$ However, it is clear that mathematics could make a huge contribution to many areas of experimental cancer investigation since there is now a wealth of experimental data which requires systematic analysis. Indeed,

\footnotetext{
* Received by the editors October 15, 2004; accepted for publication (in revised form) November 11, 2005; published electronically May 1, 2007. This work has been funded by EPSRC eScience grant titled "Integrative Biology" GR/S72023/01 and the Wellcome Trust Value in People award 070754/Z/03/Z.

http://www.siam.org/journals/sirev/49-2/44629.html

$\dagger$ Oxford Centre for Industrial and Applied Mathematics, Centre for Mathematical Biology, Mathematical Institute, Oxford University, 24-29 St. Giles', Oxford OX1 3LB, United Kingdom (roose@ maths.ox.ac.uk).

$\ddagger$ Corresponding author. Oxford Centre for Industrial and Applied Mathematics, Mathematical Institute, Oxford University, 24-29 St. Giles', Oxford OX1 3LB, United Kingdom (chapman@ maths.ox.ac.uk).

$\S$ Centre for Mathematical Biology, Mathematical Institute, Oxford University, 24-29 St. Giles', Oxford OX1 3LB, United Kingdom (maini@maths.ox.ac.uk). This author was supported by Queensland University of Technology through a QUT Visiting Fellowship.

${ }^{1}$ A search using the terms "cancer mathematical model," "cancer model," "tumor mathematical model," and "tumor model" found fewer than $5 \%$ of the 1.5 million papers identified using the term "cancer."
} 
one could argue that to exploit fully the data generated by this huge experimental effort requires such an approach [1].

There are several different stages in the growth of a tumor before it becomes so large that it causes the patient to die or reduces permanently their quality of life. There is a lot of controversy over how exactly cancer is initiated, but it is a generally accepted view that it requires several gene mutations to turn a normal cell into a cancer cell (see the recent review [2]). The factors that trigger these mutations are largely unknown, but are thought to include both environmental and hereditary effects. One of the outcomes of this series of mutations is an increase in the proliferation rate and a decrease in the death rate of the cells, giving rise to a clump of tumor cells growing faster than the host cells. However, even a fast growing clump of tumor cells cannot grow beyond a certain size, since there is a balance between cells inside the clump consuming nutrients and nutrient diffusion into the clump. ${ }^{2}$ Therefore, one of the most important steps in malignant tumor growth is angiogenesis, which is the process by which tumors develop their own blood supply. For this reason novel drugs are being developed specifically to target tumor blood vessels. Once the tumors have acquired their own blood supply, the tumor cells can escape the primary tumor via the circulatory system (metastasis) and set up secondary tumors elsewhere in the body. After angiogenesis and metastasis, the patient is left with multiple tumors in different parts of the body that are very difficult to detect and even more difficult to treat.

Because there are three distinct stages (avascular, vascular, and metastatic) to cancer development, researchers often concentrate their efforts on answering specific questions on each of these stages. This review aims to describe the current state of mathematical modeling of avascular tumor growth, i.e., tumors without blood vessels. This is not to say that this is the most important aspect of tumor growth - on the contrary, from a clinical point of view angiogenesis and vascular tumor growth together with metastasis are what cause the patient to die, and modeling and understanding these is crucial for cancer therapy. Nevertheless, when attempting to model any complex system it is wise to try and understand each of the components as well as possible before they are all put together. Avascular tumor growth is much simpler to model mathematically, and yet contains many of the phenomena which we will need to address in a general model of vascular tumor growth. Moreover, the ease and reproducibility of experiments with avascular tumors means that the quality and quantity of experimental evidence exceeds that for vascular tumors, for which it is often difficult to isolate individual effects. In particular, because some (but certainly not all) tumor cell lines grown in vitro form spherical aggregates [3], the relative cheapness and ease of in vitro experiments in comparison to animal experiments has made tumor spheroid assays very popular.

Thus we see the modeling of avascular tumors as a first step toward building models for fully vascularized tumors. In addition, there are some questions concerning avascular tumors which may be interesting in their own right, including the recent controversial hypothesis that all humans have small dormant avascular tumors in their bodies [4]. There are also parallels between avascular tumor growth and the growth of a tumor tissue in the microregion supported by a single blood vessel inside a vascular tumor, as illustrated in Figure 1, where the different regions of both avascular and

\footnotetext{
${ }^{2}$ The exact manner of the nutrient-limited growth depends of course on the specific tumor type. For example, melanomas grow on the surface of the skin as a thin layer and therefore they can grow much bigger during the avascular stage than solid tumors inside tissues. However, for one reason or another even melanomas become angiogenic and can cause metastatic tumors.
} 

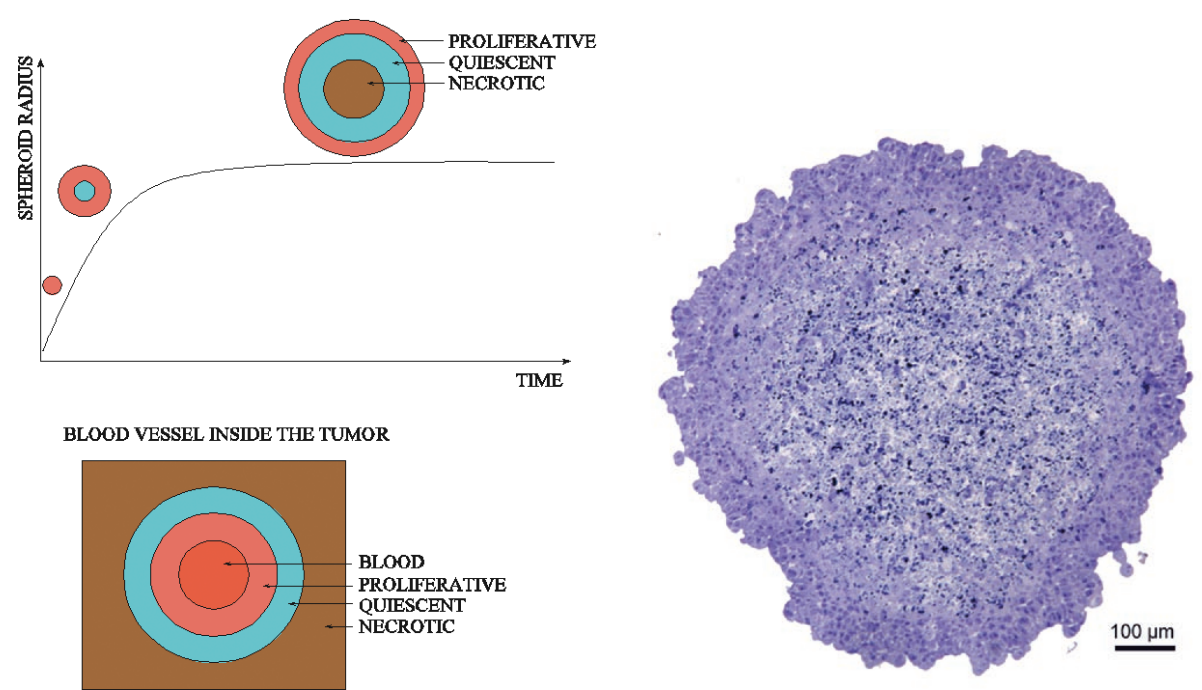

Fig. I Left: Schematic illustration showing tumor spheroid growth. Right: 1 micron of spheroid section showing the proliferative rim and the necrotic core. (Right panel reprinted from [5] with permission of the Optical Society of America.)

vascular tumors (to be explained shortly) are shown. Thus, avascular tumor modeling can be of use when making predictions and designing experiments on vascular and metastatic tumors, which are much more time consuming and difficult as they have to be performed in vivo.

The main finding of in vitro tumor spheroid experiments so far has been that the spheroids grow until they reach a critical size, when growth stops [3]. This critical size is determined by a balance between cell proliferation and cell death inside the spheroid. The main experimental observations have been that when cancer cells are in a high nutrient environment they proliferate, in low nutrient levels the tumor cells trigger cell death (apoptosis), and in intermediate nutrient levels the tumor cells stay quiescent. This translates into the schematic growth curve shown in Figure 1. The different nutrient levels inside the tumor spheroid are determined by the movement and consumption of nutrients within the tumor.

The fact that in vitro experiments clearly showed that nutrient (in particular oxygen) diffusion limits tumor spheroid growth paved the way for the angiogenesis hypothesis. This hypothesis is that, in order to grow large, tumors need to obtain their own blood vessels and therefore must recruit vessels from the host vasculature through angiogenesis [6]. This hypothesis has led to considerable research into a possible cure for cancer by identifying factors that would stop angiogenesis [7]. Currently, several clinical trials in their final stages are evaluating the efficacy and safety of antiangiogenic therapies and the scientific community is eagerly awaiting the results (see the webpage describing current clinical trials http://cancer.gov/ clinicaltrials/developments/anti-angio-table/). There are also several models describing different aspects of angiogenesis (see the recent review by Mantzaris, Webb, and Othmer [8]). However, in this review we will concentrate on the processes that take place before the commencement of angiogenesis, during the phase of avascular tumor growth. We will also not discuss models that address evolutionary questions about cancer, i.e., how and why cancer comes about and how a normal cell turns into a 
cancer cell over the course of its lifetime $[9,10,11,12,13,14,15,16,17,18,19]$, because there is a good recent review already available [2]; nor will we discuss the adaptive response of tumors, although in many cases this can be analyzed via a natural extension of the models we present. Finally, we will not discuss models describing drug delivery in this review. The mathematical modeling of drug delivery is so well established experimentally and theoretically that it itself requires a separate review; we refer the reader to the recent review by Jain [20].

The process of nutrient consumption and diffusion inside tumors has been modeled since the mid-1960s. There have been several reviews [21, 22, 23] of this area of tumor modeling published over the last few years. However, they all focus on different aspects to those we address. For example, the most recent review [21] discusses the general concepts of modeling from a historical perspective, rather than focusing on particular models. In the present review the aim is to describe some of the most widely used mathematical models and developments in the area of avascular tumor growth in more mathematical detail. Hopefully this will engage applied and pure mathematicians while not alienating the biologists and chemical engineers who work at the interface between mathematics and biology.

In this review we can clearly not describe every model of avascular tumor growth. Most models fall into two categories: (1) continuum mathematical models that use space averaging and thus consist of partial differential equations; and (2) discrete cell population models that consider processes that occur on the single cell scale and introduce cell-cell interaction using cellular automata-type computational machinery. We will discuss each of the two approaches in detail and we aim to present a comprehensive list of references for each category. In addition, in each of the categories, we will discuss one or two models in depth. We have chosen these representatives because they were published in or are frequently referenced in biological journals read by experimentalists in the area of cancer biology and in general make quantitative predictions that have been validated experimentally. This approach to choosing the representative models ensures that we are by default selecting examples that have had real impact thus far on the scientific research of cancer. However, such an approach does mean that we risk ignoring papers that have made crucial conceptual advances, or which have put forward important hypotheses which are too difficult to test experimentally at the moment, or whose significance has not yet been recognized by the experimental community. Therefore we broaden our review by summarizing these models also.

In the conclusion section a critique of the current state of mathematical modeling will be presented and possible new emerging areas of mathematical and biological research will be highlighted.

2. Continuum Cell Population Models. Mathematical models describing continuum cell populations and their development classically consider the interactions between the cell number density and one or more chemical species that provide nutrients or influence the cell cycle events of a tumor cell population. Thus these models typically consist of reaction-diffusion-convection equations. One of the best parameterized of these models is due to Casciari, Sotirchos, and Sutherland [24], and will be described in the first part of this section. In the second part of this section we describe models from a relatively new and emerging area of avascular tumor modeling that deals with modeling of the mechanical interaction between the tumor cells and their surroundings.

Cell Population and Nutrient Consumption Model. The paper that first proposed that diffusion and nutrient consumption might be limiting solid tumor growth 
was probably Burton [25], and since then a large number of studies have described the spatio-temporal interactions between tumor cell populations and nutrients [22], [24]-[82]. Early models of nutrient-limited tumor growth calculated the nutrient concentration profiles as a function of tumor spheroid radius that was changing due to the rate of cell proliferation $[25,29,44,49,50,58,59,66,72]$. The later models have incorporated differing degrees of complexity for cell movement. For example, cells can be considered to move in either a convective manner [37, 45, 62, 67, 79] or actively in a diffusive manner $[65,71,75,77,80]$, or in a diffusive/chemotactic manner [56, 62, 65]. Most models consider tumor cell proliferation and death to be dependent on only one generic nutrient (most often oxygen). However, some consider the effect of several nutrients and $\mathrm{pH}$ on the cell population [24, 83]. While the details of any particular model in a specific experimental context are different, the fundamental underlying principle behind these models can be captured in the following short statement (see also Figure 1):

Tumor cells consume nutrients. Nutrients diffuse into the tumor tissue from the surrounding tissue. Therefore, if the tumor is very large, the nutrients cannot reach all parts of the tumor tissue. This leads to a decrease in tumor cell proliferation and eventual cell death in regions lacking nutrients. The steady size of the tumor spheroid is reached when the cell proliferation in regions rich in nutrients balances cell death in regions poor in nutrients.

As the models which treat both diffusion/consumption of nutrients and cell movement, rather than solely nutrient diffusion/consumption, are more biologically relevant we wish to discuss one of these models as a representative of the class. One can find very few experimentally validated models for simultaneous cell population and nutrient interaction. Of these, we have chosen Casciari, Sotirchos, and Sutherland [24] because it is a very good example of the class and it includes a sufficient level of biochemical complexity for it to be experimentally relevant. This model considers a spherical tumor and the interaction of tumor cells with oxygen, glucose, lactate, carbon dioxide, and bicarbonate, chloride, and hydrogen ions, and it aims to answer quantitative questions on the expected $\mathrm{pH}$ inside tumors.

Equations for Chemical Species Inside the Spheroid. The equations describing the distribution of molecular species inside the tumor spheroid are classical transport/mass conservation equations. Figure 2 shows the schematic model for $\mathrm{pH}$ regulation inside the tumor cells [24]. The conservation equations for the different chemical species are

$$
\frac{\partial C_{i}}{\partial t}+\nabla \cdot \mathbf{N}_{i}=P_{i}
$$

where $C_{i}$ are the concentrations of the chemical species, subindex $a$ for oxygen, $b$ for glucose, $c$ for lactate ion, $d$ for carbon dioxide, $e$ for bicarbonate ion, $f$ for chloride ion, and $g$ for hydrogen ion concentration; $\mathbf{N}_{i}$ is the flux of each of the chemical species inside the tumor spheroid; and $P_{i}$ is the net rate of consumption/production of the chemical species both by the tumor cells and due to chemical reactions with other species.

For uncharged molecules, i.e., glucose, oxygen, and carbon dioxide, the flux is given by Fick's law,

$$
\mathbf{N}_{i}=-D_{i} \nabla C_{i},
$$

where $D_{i}$ are (positive) constant diffusion coefficients. 


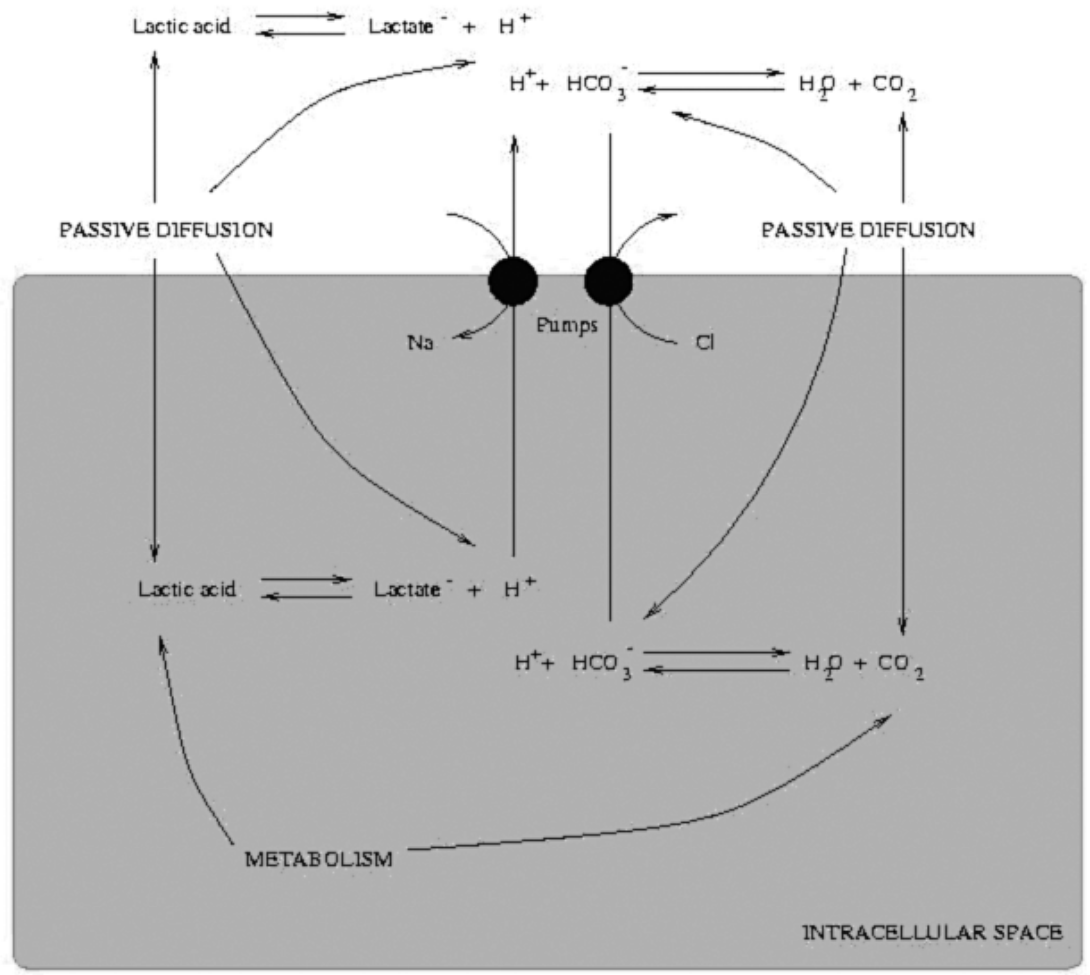

Fig. 2 A model for $p H$, oxygen, and glucose regulation within the cell and its surroundings, after Casciari, Sotirchos, and Sutherland [24]. (Reprinted with permission of Blackwell Publishing.)

The flux of ionic species, i.e., lactate, bicarbonate, and hydrogen ions, in dilute solution must take into account both electric field driven charge migration and diffusion, and is given by

$$
\mathbf{N}_{i}=-z_{i} u_{i} F C_{i} \nabla \Phi-D_{i} \nabla C_{i}
$$

where $z_{i}$ is the ionic charge of species, $u_{i}$ is the mobility, $F$ is Faraday's constant, and $\Phi$ is the electrical potential. For dilute solutions Casciari, Sotirchos, and Sutherland [24] took $u_{i}$ to be given by the Nernst-Einstein equation

$$
u_{i}=D_{i} / R_{g} T,
$$

where $R_{g}$ is the gas constant, $T$ is the absolute temperature, and $D_{i}$ is the diffusion constant. By applying the assumption that there is zero net electrical current, so that $\sum_{k} z_{k} \mathbf{N}_{k}=\mathbf{0}$, (3) can be rewritten in the form

$$
\mathbf{N}_{i}=-D_{i}\left(\nabla C_{i}-z_{i} C_{i} \frac{\sum_{k} z_{k} D_{k} \nabla C_{k}}{\sum_{k} z_{k}^{2} D_{k} C_{k}}\right) .
$$

Thus, the flow of a specific ionic species depends on the concentration gradients of all the ionic species present in the tissue. 
Finally, by considering the breakdown of glucose through glycolysis and the Krebs cycle $^{3}[84]$ and the detailed metabolic pathway for $\mathrm{pH}$ regulation (see Figure 2 for schematic representation) on a single cell level, Casciari, Sotirchos, and Sutherland [24] arrived at the following terms describing the consumption of oxygen and glucose by the tumor cells and interactions between the other chemicals and ionic species in this cascade:

$$
\begin{aligned}
& P_{a}=-\Omega\left(A_{a}+\frac{B_{a}}{C_{b} C_{g}^{m}}\right)\left(\frac{C_{a}}{C_{a}+K_{m a}}\right), \\
& P_{b}=-\Omega\left(A_{b}+\frac{B_{b}}{C_{a}}\right)\left(\frac{1}{C_{g}^{n}}\right)\left(\frac{C_{b}}{C_{b}+K_{m b}}\right), \\
& P_{c}=-\left(2 P_{b}-P_{a} / 3\right), \\
& P_{d}=-k_{f} C_{d}+k_{r} C_{e} C_{g}, \\
& P_{e}=k_{f} C_{d}-k_{r} C_{e} C_{g}-P_{a}, \\
& P_{f}=0 \\
& P_{g}=k_{f} C_{d}-k_{r} C_{e} C_{g}-P_{a}+P_{c},
\end{aligned}
$$

where $\Omega$ is the number of cells per unit volume of spheroid, assumed to be constant. In (6) the relations for $P_{c}, P_{d}, P_{e}, P_{f}$, and $P_{g}$ were derived using the detailed consideration of the Krebs cycle, and the parameters $k_{f}$ and $k_{r}$ are determined from the detailed chemical reaction schemes. The functional forms of oxygen consumption $P_{a}$ and glucose consumption $P_{b}$ were derived empirically from experiments. In particular, independent measurements of (a) oxygen consumption at different levels of glucose and $\mathrm{pH}$, and (b) glucose consumption at different levels of oxygen and $\mathrm{pH}$ were conducted and the empirical functional forms in (6) were fitted to the data. Our understanding is that this fitting was done by eye, rather than through a statistical analysis (as is common in mathematical biology, since often only a few data points are available when estimating a nonlinear curve). Thus the functional forms for oxygen and glucose consumption should be considered representative, rather than cast in stone - many other functional forms could be chosen. Indeed, it is almost certain that different cancer cell lines would have different oxygen and glucose consumption rates, and it is not clear that they can all be approximated by the functional forms in (6); each would have to be assessed individually against available experimental data. Moreover, even Casciari, Sotirchos, and Sutherland themselves used a different functional form at low levels of oxygen and glucose, where they set [24]

$$
\begin{aligned}
& P_{a}=-\Omega\left(\phi_{a}-\beta_{a} C_{b}^{2}\right) \frac{C_{a}^{2}}{C_{a}^{2}+\varsigma_{a}} \frac{C_{b}^{2}}{C_{b}^{2}+\varsigma_{b}}, \\
& P_{b}=-\Omega\left(\phi_{b}-\beta_{b} C_{a}^{2}\right) \frac{C_{b}^{2}}{C_{b}^{2}+\varsigma_{b}},
\end{aligned}
$$

where $\phi_{a}, \beta_{a}, \varsigma_{a}, \phi_{b}, \beta_{b}, \varsigma_{b}$ are new fitting parameters which are again determined by comparison with experimental data.

The chemical species conservation equations require boundary conditions. Casciari, Sotirchos, and Sutherland [24] assumed that on the boundary of the spheroid,

\footnotetext{
${ }^{3}$ The Krebs cycle is a sequence of 10 biochemical reactions, brought about by mitochondrial enzymes, that involves the oxidization of a molecule of acetyl-CoA to two molecules of carbon dioxide and water. Each turn of the cycle can result in the formation of 12 molecules of ATP (direct source of energy for cells) per molecule of acetyl-CoA [84].
} 
$r=R$, there is a flux of chemical species given by the difference in concentration of chemical species in the bulk medium outside the spheroid $\left(C_{i, \text { out }}\right)$ and concentration at the boundary of the spheroid, i.e.,

$$
\left.\mathbf{e}_{r} \cdot \mathbf{N}_{i}\right|_{r=R}=K_{i}\left(C_{i, \text { out }}-C_{i}\right),
$$

where $K_{i}$ is the mass transfer coefficient of species $i$, and $\mathbf{e}_{r}$ is the unit vector in the radial direction. The coefficients in (7) were measured using a Bellco spinner flask, and the values were found to depend on the diffusivity of the molecule in question, viscosity of the culture medium, and spinner flask characteristics (the exact experimental setup is described in [85]). The boundary condition (7) is equivalent to Newton's law of cooling and assumes that the culture medium is well mixed. If this is not the case, then it may be necessary to solve for the concentration of species in the culture also, imposing continuity of concentration and flux across the spheroid boundary.

In the case of spherical symmetry we also impose

$$
\left.\nabla C_{i}\right|_{r=0}=\mathbf{0},
$$

so that there is no singularity in the concentrations at the origin.

Equations for Cell Movement and Proliferation. The spheroid growth equations proposed in [24] are based on the law of mass conservation, so that the velocity of cell movement $\mathbf{v}$ is given by

$$
\nabla \cdot \mathbf{v}=\lambda F\left(C_{i}\right)
$$

where $\lambda$ is the maximum rate of cell proliferation and $F\left(C_{i}\right)$ is the scaling function for the nutrient/chemical species dependent proliferation. To find the functional form of $F\left(C_{i}\right)$ Casciari, Sotirchos, and Sutherland [24] conducted a series of experiments to measure the cell population dynamics in cell monolayer cultures at different levels of $\mathrm{pH}$ and oxygen and glucose concentration. Monolayer cultures are a good experimental tool for determining reaction kinetics and cell proliferation characteristics, since nutrient and other chemical concentrations can be precisely controlled without spatial variation. Of course, cell signaling cues arising from cell-cell contact may be different in monolayers and spheroids, but such effects are taken to be secondary and are not included in the model. Casciari, Sotirchos, and Sutherland [24] fitted the data with the following empirical function describing the rate of cell proliferation in different chemical environments:

$$
F\left(C_{i}\right)=G_{1}\left(\frac{C_{a}}{G_{a}+C_{a}}\right)\left(\frac{C_{b}}{G_{b}+C_{b}}\right)\left(\frac{1}{C_{g}}\right)^{n}
$$

where the $G_{i}$ 's are fitting parameters. Again, this functional form should be taken as representative rather than set in stone. One limitation of the functional form (10) is the fact that the cell proliferation term $F$ is always strictly positive. This implies that for the EMT6/Ro spheroids considered in [24] there can never be a necrotic core, that is, a region where there is only cell death. Since the cells are proliferating everywhere there cannot be a steady state for the tumor radius. Thus this model is applicable for transient, pre-plateau phase spheroids. To model growth saturation and necrosis fully we would need to include a cell death term in the right-hand side of (9). This would make the model parameterization much more difficult since in addition to measuring nutrient-dependent cell proliferation, one would also need to simultaneously measure 
cell death, which can in principle also depend on the levels of metabolites inside the spheroid.

Equation (9) implies that the cells are incompressible and there is no change in cell density at any given point in space, so that as a cell proliferates the cells surrounding it move accordingly to accommodate the new arrivals. These assumptions are probably reasonable for spheroids grown in free suspension culture, but are more questionable in a confined environment.

In the symmetrical case of a spherical tumor we may suppose that the cells move radially so that there is only one nonzero velocity component, which depends only on the distance from the spheroid center, $r$, and time. In this case (9) is sufficient to determine the movement of the tumor cells and the spheroid boundary. However, in any nonsymmetric situation conservation of mass alone is not enough to determine the cell velocity, and some form of force balance, along with an additional constitutive relation, needs to be considered. Such an extension complicates the model considerably and leads to difficult questions concerning continuum mechanics in growing media. These are seen by most biologists as peripheral to the central biochemical nature of cancer. While this is undeniable, in the absence of symmetry it is difficult to see how to close a biochemical model such as (1)-(9) without some discussion of mechanics. We will return to this question later when we discuss generalizations of the model of Casciari, Sotirchos, and Sutherland [24], but for the moment we continue to make the assumption of radial symmetry.

By solving (9) with spherical symmetry we find that the radial cell velocity $v_{r}$ at a point inside the spheroid and the evolution of the tumor boundary $R$ are given by

$$
\begin{aligned}
v_{r}(r) & =\lambda \frac{1}{r^{2}} \int_{0}^{r} F\left(C_{i}\right) d \bar{r} \\
\frac{d R}{d t}=v_{r}(R) & =\lambda \frac{1}{R^{2}} \int_{0}^{R} F\left(C_{i}\right) d \bar{r} .
\end{aligned}
$$

Equations (1)-(11) form a closed system and can be solved numerically or further simplified to enable approximate analytic solutions to be obtained.

We stress that while the model by Casciari, Sotirchos, and Sutherland [24] includes several phenomenological functions and parameters, it is one of the best parameterized models, and the relatively small number of phenomenological constants makes it possible to compare the results of the model to experiments. Using the model Casciari, Sotirchos, and Sutherland [24] predicted that the oxygen and glucose concentrations will fall in the middle of the spheroid, resulting in a significantly reduced rate of proliferation. This gives rise to the viable rim, that is, a region of high cell proliferation near the edge of the spheroid. The width of the viable rim can be measured experimentally using immunohistochemical techniques to stain the cells at specific checkpoints in the cell cycle. Casciari, Sotirchos, and Sutherland [24] reported that the experimentally measured width of the viable rim is in agreement with that predicted by the model, although it should be noted that the experiments involved a limited number of trials and gave a mean width of the viable rim $(7 \pm 23) \%$ greater than that predicted by the model; the large spread in the result was due to the high variability in the experimental data. The model also predicts that the $\mathrm{pH}$ inside the tumor spheroid should be different from that in the external medium, with higher acidity at the tumor center than near the tumor boundary. This quantitative prediction has also been confirmed experimentally [24]. 


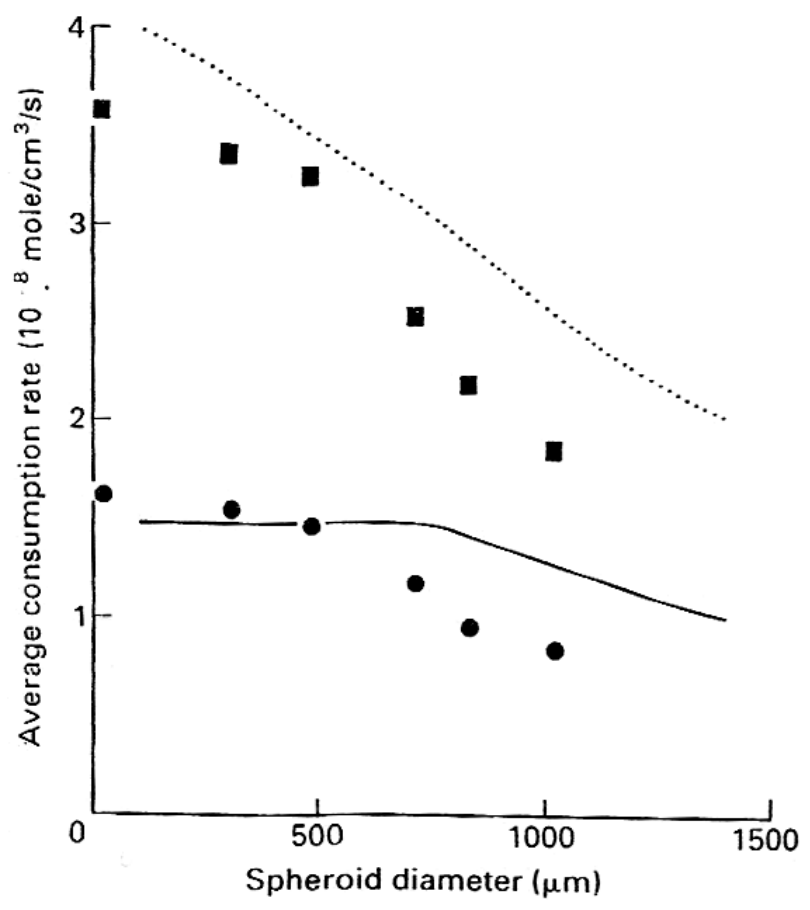

Fig. 3 Comparison between Casciari, Sotirchos, and Sutherland [24] model prediction (lines) and the experimentally measured (symbols) values for glucose (squares and dotted line) and oxygen (circles and solid line). (Reprinted with permission of Blackwell Publishing.)

The study of Casciari, Sotirchos, and Sutherland [24] is an example of the success of mathematical modeling in tumor biology (see, for example, Figure 3 for a comparison between the predicted and experimental measurements of oxygen and glucose consumption), but it also highlights some of the dangers. Even with such a well-parameterized model, the quantitative agreement of model prediction and experiment is only satisfactory (far worse than could be expected in physics, for example), and the experiments themselves show a huge variation. This highlights the need for accurate measurements in order to generate accurate parameter estimates, but also warns against placing too much emphasis on fitting models to data - what is more important is to get the biochemistry correct qualitatively (which, of course, is what Casciari, Sotirchos, and Sutherland were trying to do when they chose functional forms such as (10)), and to be able to predict general features of the solution (such as higher acidity at the tumor center) reliably.

There is a delicate balance when building any model between reliability and realism. As more and more is discovered about the detailed biochemistry of tumors we have to guard against the tendency to develop overly complex or detailed mathematical models for which we cannot hope to realistically or reliably estimate all the parameter values. While such models may seem sophisticated, their predictions are likely to be less accurate than a better-parameterized simpler model. One of the advantages of mathematical modeling in this regard is its ability to determine what qualitative details are sacrificed when simpler models are used. 
Generalizations of Cell Movement and Proliferation Equation. Several modifications of the cell proliferation equations (9) and (10) have been presented in the literature. We outline the main approaches below. The most general modification of the model of Casciari, Sotirchos, and Sutherland [24] would be to take into account explicitly that the tumor comprises cells, interstitial fluid, and possibly extracellular matrix, and to treat the tumor as a multiphase material with these as the different phases. The model will then need to describe the mechanical and biochemical interactions that take place between all the phases considered. If we include random cell migration and cell death, each phase can be described by

$$
\frac{\partial \Phi_{i}}{\partial t}+\nabla \cdot\left(\mathbf{v}_{i} \Phi_{i}\right)=\nabla \cdot\left(D_{i} \nabla \Phi_{i}\right)+\lambda_{i}\left(\Phi_{i}, C_{i}\right)-\mu_{i}\left(\Phi_{i}, C_{i}\right),
$$

where, for phase $i, \Phi_{i}$ is the volume fraction $\left(\sum_{i} \Phi_{i}=1\right), \mathbf{v}_{i}$ is the velocity, $D_{i}$ is the random motility or diffusion, $\lambda_{i}\left(\Phi_{i}, C_{i}\right)$ is the chemical and phase dependent production, and $\mu_{i}\left(\Phi_{i}, C_{i}\right)$ is the chemical and phase dependent degradation/death. The most common models consider only the cell phase (as in Casciari, Sotirchos, and Sutherland [24]), or cell and interstitial fluid phases.

The simplest version of (12), which has been used extensively, is a two phase model with the two phases being live cells and dead cells $[67,68]$. If we know the average volume of the live and dead cells, $V_{L}$ and $V_{D}$, respectively, then the volume fraction equations can be converted into equations for the live and dead cell number density $n$ and $m$, respectively, giving

$$
\begin{aligned}
\frac{\partial n}{\partial t}+\nabla \cdot\left(\mathbf{v}_{n} n\right) & =\nabla \cdot(D \nabla n)+\lambda\left(n, C_{i}\right)-\mu\left(n, C_{i}\right), \\
\frac{\partial m}{\partial t}+\nabla \cdot\left(\mathbf{v}_{m} m\right) & =\mu\left(n, C_{i}\right),
\end{aligned}
$$

where, of course, dead cells have no random motion. Usually $D$ is constant, but occasionally (13) is modified to include nonlinear diffusion of tumor cells $[65,76,77]$ by setting $D=D\left(n, C_{i}\right)$. Most models consider only one chemical species (usually oxygen or some generic nutrient), so that the biochemistry is much simpler than that of Casciari, Sotirchos, and Sutherland [24]. It is common to assume that the convective movement of both cell types is the same, so that $\mathbf{v}_{n}=\mathbf{v}_{m}=\mathbf{v}$, say [67,68]. Then, combining (13) with the volume conservation relation $n V_{L}+m V_{D}=1$, we obtain an expression for the cell velocity,

$$
\nabla \cdot \mathbf{v}=\nabla \cdot(D \nabla n) V_{L}+\lambda\left(n, C_{i}\right) V_{L}-\mu\left(n, C_{i}\right)\left(V_{L}-V_{D}\right),
$$

which is the analogue of (9). In the case of radial symmetry this is again enough to determine $\mathbf{v}$, without needing to worry about force balances.

In principle, the multiphase approach can be extended to include three or even more phases (including different cell subpopulations, such as cancer and normal cells, or cells with and without a certain gene, or even drug carrying microbeads, etc.). However, it has to be noted that with the addition of each phase the parameterization of the model becomes more and more difficult, since large numbers of independent experiments are needed to validate the model. The general multiphase model (12) needs to be supplemented by equations describing the velocity of each phase. The usual practice is again to assume that all phases move with the same velocity, so that with the further assumption of radial symmetry, conservation of mass allows the 
velocity to be found without worrying about constitutive relations and force balances. However, there seems to be no reason for the velocities of the components to be the same or even of the same sign, especially when there is transfer between the phases. When the assumption of radial symmetry is dropped, so that force balances need to be considered to close the model anyway, there seems to be no advantage in assuming that all the velocities are the same, and they may be better approximated by using constitutive laws for each phase and accounting for momentum transfer between phases. We will describe this approach in the next section when we consider mechanochemical models of tumor growth.

Several mathematicians have analyzed generic nutrient-limited tumor spheroid growth equations with the aim of understanding qualitatively the sort of behaviors that can arise as a result of different biological effects. For example, Burkowski [86] and Byrne [87] analyzed the effect of the incorporation of time delays in the cell proliferation and death terms in a generic nutrient-limited, spheroid growth model. This approach results in delay differential equations that the authors hypothesized are able to describe the oscillatory growth of tumor spheroids. Byrne and Gourley [35] considered what effect growth factors influencing signal transduction pathways of tumor cells have on generic nutrient-limited spheroid growth. Chaplain, Ganesh, and Graham [80] hypothesized that the prepatterning of growth inhibitors and growth promoters on the tumor surface by a reaction-diffusion mechanism involving two unidentified chemical species could explain the asymmetries that arise during tumor growth.

An important set of experiments which give information about the movement of cells in tumor spheroids was conducted by Dorie et al. [88, 89]. The experiments involved attaching labeled tumor cells (in both homotypic and heterotypic combinations) and inert polystyrene microspheres to the outside of EMT6 cultured tumor spheroids. The results showed that while the distribution of labeled cells or microspheres always spread out (as it would due to random motion), if the labeled cells were the same type as the tumor spheroid, then the maximum of the distribution remained at the spheroid edge, while labeled cells heterotypic with the spheroid and inert microbeads migrated towards the center of the spheroids, a process known as internalization. Even when tumor cell proliferation was stopped by irradiation, this internalization was still seen. These experiments have been modeled by several authors using multiphase continuum models $[60,62,76]$. Some of these models include active cell migration to nutrient rich areas, with the microspheres forced into the center of the tumor as the cells squeeze past them; others explain the motion of the microspheres using only passive cell motion, as the cells at the center die and the outer cells replace them, carrying the microspheres with them. It is still not clear which, if either, of these effects is responsible for the internalization process.

Several authors have modeled the invasion of tumors into other tissue using traveling-wave solutions of diffusion-based tumor-cell-population models [55, 56, 65, $73,75]$. Sherratt [65] performed a traveling-wave analysis of a nonlinear diffusion model for the interaction of tumor cells and the extracellular matrix with the aim of proposing a mechanism for the formation of connective tissue capsules around avascular tumors. These capsules around solid tumors are highly collagenous layers of connective tissue surrounding the tumor and are most commonly found in large vascular tumors. Marchant, Norbury, and Perumpanani [55] analyzed a model for tumor cell migration and interaction with connective tissue and protease. Using a traveling-wave analysis they were able to identify a range of possible behaviors, such as the development of spatial heterogeneity, traveling waves with wavy tails, etc. In particular, their models address the question of how the tumor cell interaction with 
connective tissue and protease influences tumor growth. They found that under certain circumstances tumor cell profiles, as well as connective tissue profiles, can advance or recede like traveling waves.

As set out above there are a relatively large number of continuum cell population and nutrient consumption models. However, very few of them have been well parameterized and tested against experimental data. While experimental validation is undoubtedly crucial to the success of any model, phenomenological models can provide a framework in which to think about the physical system, allow us to explore the effects of different hypotheses, advance our conceptual understanding, and even make predictions. An example of the latter is given by the model by Gatenby and Gawlinski [90] which describes the transition from benign to malignant growth using the acidmediated invasion hypothesis. It consists of three coupled reaction-diffusion equations for cancer cells, normal cells, and acidity and explores the hypothesis that, as a result of anaerobic metabolism, cancer cells create an acidic environment which kills normal cells. The model predicts the existence of an interstitial gap at the tumor-host interface and this prediction has been confirmed experimentally (see Figure 4). This example shows the power of an interdisciplinary approach and illustrates its potential. It is unfortunate that many model predictions are not tested experimentally.

Tissue Mechanics Models. Tissue mechanics models consider the mechanical interactions between tumor cells with the aim of answering questions about how the mechanical properties of the tumor, and the tissue in which the tumor grows, influence tumor growth. Since the late 1970s there have been several models that in one way or another have introduced the concept of pressure or force between tumor cells [36, $37,39,40,43,47,50,52,60,62,63,64,92,93,94,95,96,97,98,99,100,101,102$, 103]. In addition, there have been a number of papers which have proposed ways to include the effects of mechanical interaction between tumor cells into the existing mathematical framework of nutrient-limited spheroid growth. This research coincided with a conceptual change in cell biology away from a picture of cells as simple fluid bags to cells having some structural integrity, which was brought about by experimental evidence showing that cells have microskeletons which give them mechanical strength. A theoretical framework for understanding these microskeletons was pioneered by Ingber, who introduced the concept of tensegrity structures to biology (Figure 5), which are mechanical systems that are stabilized by continuous tension. One example of this is the human body, which is stabilized and controlled by tension in muscles and ligaments. However, this approach to modeling the mechanical properties of cells is in its infancy (we know of only one paper [104], which deals with a very simplified case), although it might become more popular in the future. Most models of cell tissue mechanics assume some bulk constitutive relation between stress and strain/strain rate, averaged over many cells.

The early models built on the nutrient diffusion/cell proliferation models discussed in the previous section by introducing a pressure inside the tumor. An example is the model by Greenspan for avascular tumor growth [50], which was further developed by Byrne and Chaplain [92]. In this model the diffusion of a single, generic nutrient $c$ is considered to be in pseudoequilibrium in time, so that inside the tumor spheroid $\nabla^{2} c-\lambda(c)=0$, where $\lambda(c)$ is the rate of nutrient consumption by the tumor cells. As in the previous section, mass conservation of incompressible tumor cells results in a tumor cell velocity $\mathbf{v}$ given by $\nabla \cdot \mathbf{v}=S(c)$, where $S(c)$ is the nutrient-concentration-dependent net cell proliferation rate. The authors introduce the concept of the spheroid internal pressure $p$, and relate it to the cell velocity $\mathbf{v}$ by 


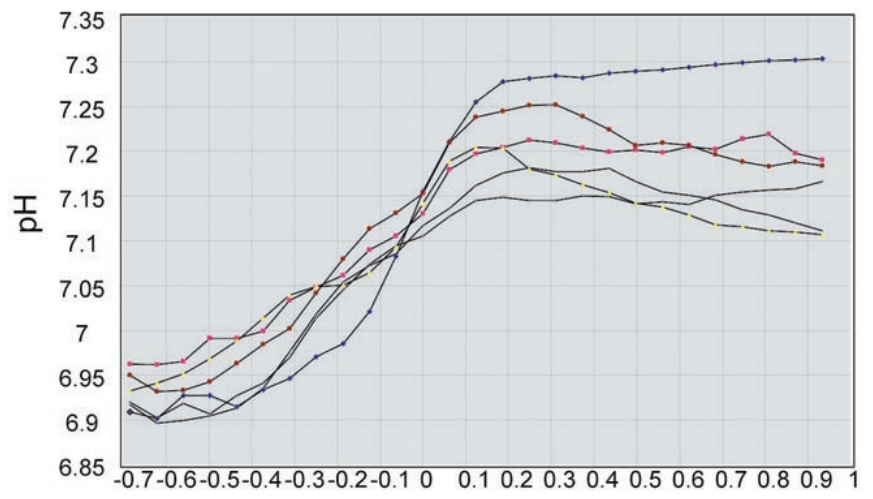

Distance from the tumor edge $(\mathrm{mm})$

(a)

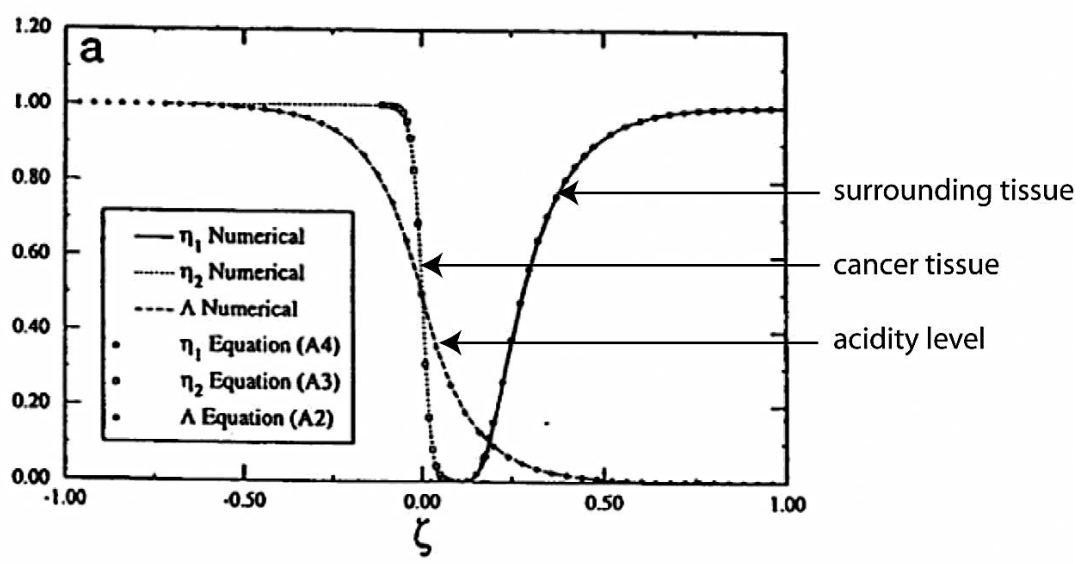

(b)

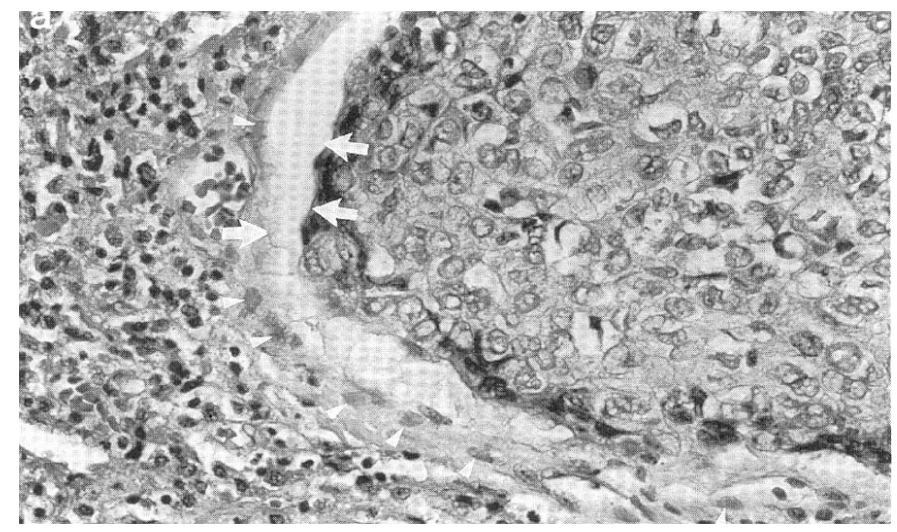

(c)

Fig. 4 (a) Experimental data showing $p H$ gradients as a distance from the tumor edge. Data shown for different angles drawn from tumor center [91]. (b) Mathematical modeling results showing intratumoral acid gradients and acid-mediated acellular gap at the tumor host interface. (c) Histopathological image of a cellular gap at the tumor-host interface in the head and neck cancer. (All figures courtesy of Dr. R. A. Gatenby and reprinted with permission of the American Association for Cancer Research.) 


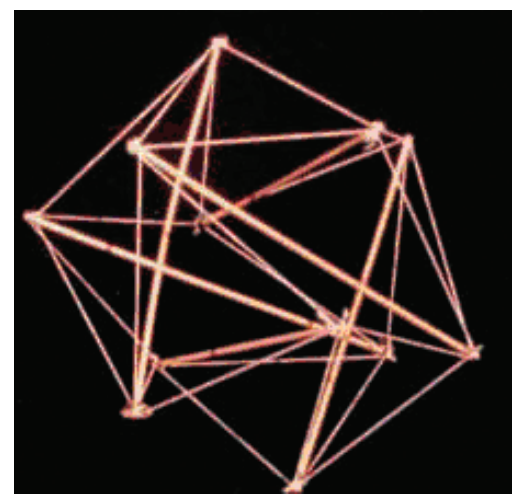

(a)

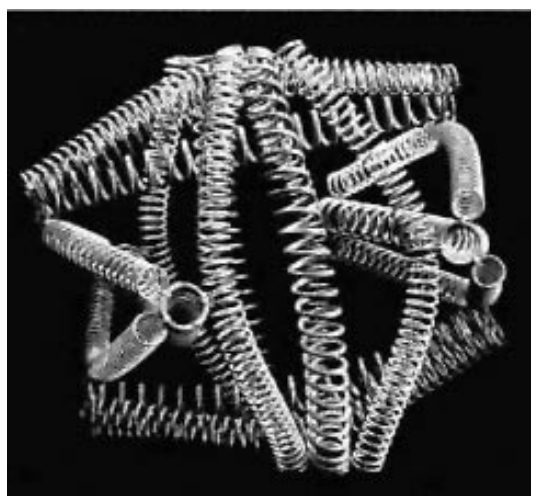

(b)

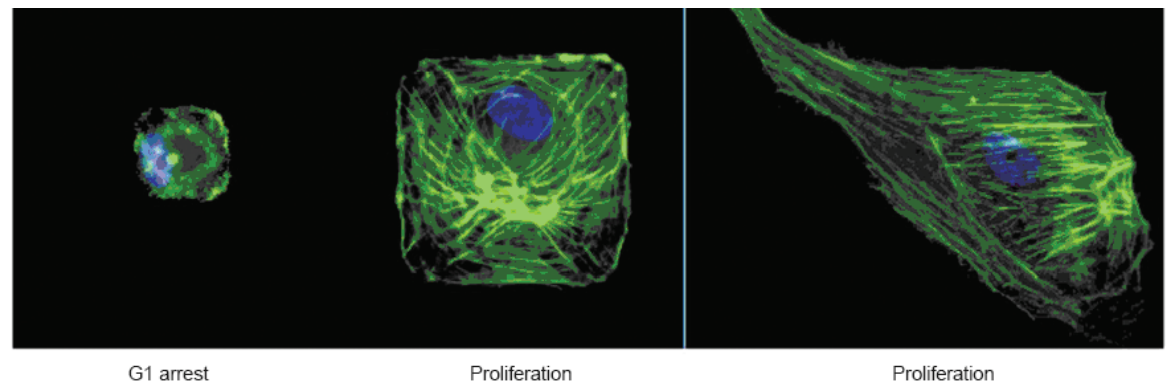

(c)

Fig. 5 Tensegrity structures from Ingber [142] (a), (b) and Huang and Ingber [143] (c). (Reproduced with permission of the Company of Biologists.)

introducing a constitutive equation

$$
\mathbf{v}=-\mu \nabla p
$$

where $\mu$ is a positive constant describing the viscous-like properties of tumor cells. This constitutive equation can be interpreted in two different ways. The first interpretation is as an overdamped force balance, in which the force on a cell maintains its velocity rather than accelerates it. The second interpretation says that the tumor tissue is "fluid-like" and that tumor cells flow through the fixed extracellular matrix like flow through a porous medium, obeying Darcy's law. Whatever the interpretation, this constitutive relation is used to derive the equation for spheroid internal pressure, $-\mu \nabla^{2} p=S(c)$. The model is closed by giving boundary conditions on the (moving) tumor boundary, $\Gamma(\mathbf{r}, t)=0$, say. The equation of motion for any boundary point is given by $\mathbf{n} \cdot \frac{d \mathbf{r}}{d t}=\mathbf{v} \cdot \mathbf{n}=-\mu \nabla p \cdot \mathbf{n}$, where $\mathbf{n}$ is the unit outward normal.

The pressure on the tumor boundary is taken to be equal to the external pressure (Greenspan [50] included surface tension effects so that the pressure is proportional to the mean curvature on the tumor boundary). The second boundary condition is on the nutrient concentration $c$. Byrne and Chaplain [92] hypothesized that energy is expended in maintaining the cell-cell bonds on the tumor surface with the requisite amount of energy increasing with the local curvature of the boundary. Assuming that the nutrient acts as a source of energy for cell-cell adhesion, the authors took the concentration of nutrient at the tumor boundary to be given by the Gibbs-Thomson 
relation, i.e., $c=c_{\infty}(1-2 \gamma \kappa)$, where $\kappa$ is the mean curvature of the tumor surface, $\gamma$ is the surface tension of the tumor surface, and $c_{\infty}$ is the nutrient concentration in the culture medium outside the spheroid.

In summary, the model equations are

$$
\begin{aligned}
& \nabla^{2} c-\lambda(c)=0, \quad \mu \nabla^{2} p+S(c)=0 \text { inside } \Gamma(\mathbf{r}, t),=0, \\
& \mathbf{n} \cdot \frac{d \mathbf{r}}{d t}=-\mu \nabla p \cdot \mathbf{n} \quad \text { on } \Gamma(\mathbf{r}, t)=0, \\
& c=c_{\infty}(1-2 \gamma \kappa), \quad p=p_{\infty} \text { on } \Gamma(\mathbf{r}, t)=0,
\end{aligned}
$$

together with initial conditions.

This model raises a number of interesting mathematical and experimental points. The first point to note is that in the case of radial symmetry the constitutive equation (15) is not needed. The model is very similar to (a simplified version of) (1)-(11) of the previous section (except for the boundary condition on $c$ at the tumor surface), and as before conservation of mass is enough to determine $v_{r}$. However, the constitutive relation (15) and the boundary condition $p=p_{\infty}$ at the tumor boundary enable us to determine the velocity of the cells when we do not have radial symmetry. Such an approach would be one way to close the model (1)-(11) when we do not have radial symmetry, and is probably the simplest way we could think of doing so.

The second new feature of (16) is the hypothesized Gibbs-Thomson relation in the nutrient boundary condition. This seems a much more speculative addition, but it may be possible to check its veracity since experimental techniques for measuring the strength of cell-cell adhesion in different nutrient environments are available. One note of caution when moving away from radially symmetric solutions is sounded if we set $\gamma=0$, in which case Byrne and Chaplain [92] found that the radially symmetric steady state is unstable to small asymmetric perturbations, with the growth rate of the most unstable mode tending to infinity as the wavelength tends to zero, so that the model seems to be ill-posed in this case. This illustrates some of the difficulties in determining simple but sensible constitutive relations for tumor tissue mechanics. Further analyses of the model, including a weakly nonlinear stability analysis, were conducted in [39, 40, 92]. Other modifications of the early cell mechanics models include the effect of growth inhibitors on the development of avascular tumors [37].

These models have given us insights into how various hypothesized mechanical interactions affect nutrient-limited tumor growth. Comparing the predictions of such models to experimental growth profiles will help to determine what sort of mechanical interactions we should be including. If this first hurdle is passed, to realize the model's full predictive potential it needs to be carefully parameterized. While experimental values are available for several of the parameters, others are difficult to measure. For example, the in vivo measurement of a pressure that is probably very low $(\sim 10$ $\mathrm{mmHg})$ in a sample that is very small in size $(\max 1 \mathrm{~mm})$ is technically very difficult. An important role of modeling in this respect is to determine, via sensitivity and/or bifurcation analysis, on which parameters the behavior of the model crucially depends, thereby identifying which parameters need to be measured accurately.

While direct pressure measurements have yet to be performed, the landmark experiments conducted by Helmlinger et al. [105] mean that there is experimental evidence which could be used to test tumor tissue mechanics models. Helmlinger et al. [105] grew tumor spheroids in different stiffness agarose gels and saw that the strength of the agarose gel influences the growth of the tumor spheroid, i.e., the stiffer the gel the smaller the tumor radius during the plateau phase of growth. Since cells 
can neither attach themselves to agarose nor degrade the agarose, Helmlinger et al. [105] concluded that solid stress inhibits tumor growth. More fundamentally, tumors in stiffer organs (bone, for example) should grow slower than tumors in very soft organs (the breast, for example), a concept that appears to make sense in the clinical setting. Helmlinger et al. [105] estimated the solid stress that might be generated during spheroid growth by measuring the mechanical properties of agarose gels, fitting a nonlinear hyperelastic strain energy function to the experimental data, and using this strain energy function to calculate the stress that is needed to move the tumor boundary from the initial one-cell radius to the final plateau-phase radius. However, as pointed out by Chen, Byrne, and King [97], the strain energy function used by Helmlinger et al. [105] does not satisfy the condition of no stress at no strain. Furthermore, reported values of the bulk modulus of the LS174T tumor used by Helmlinger et al. [105] and measured by the same group of authors [106] are in the range of $30 \pm 10 \mathrm{mmHg}$. Thus, if the stress within the spheroid was of order of $100 \mathrm{mmHg}$ as claimed in [105], then upon the release of the spheroid from the agarose, the spheroid should swell to two to three times its prerelease size. This experiment of releasing the spheroid from the agarose confinement was actually carried out by the original authors and no large swelling was seen. Instead it was noted that the rate of spheroid growth was recovered after release, indicating that the solid stress actively influenced the cell cycle instead of passively compressing the tumor tissue. It is therefore likely that the stress in spheroids grown in agarose is actually lower than $100 \mathrm{mmHg}$, as reported in [105].

Despite this controversy concerning the strain energy function, the paper by Helmlinger et al. [105] has motivated several researchers to model this experimental situation in more detail $[93,95,97,107]$. Three of these papers are theoretical studies, while Roose et al. [93] presents a combined theoretical and experimental study with an aim to correct the quantitative estimate of the solid stress inside the tumor spheroids by considering a linear poroelasticity model where the tissue growth has been absorbed into the stress-strain relation in a manner similar to thermal elasticity as used in classical solid mechanics [108]. They used the following constitutive equation linking effective tissue stress to tissue strain and fluid pressure:

$$
\sigma_{i j}=2 G \varepsilon_{i j}+\left(K-\frac{2}{3} G\right) \varepsilon_{k k} \delta_{i j}-p \delta_{i j}-K \eta \delta_{i j}, \quad \varepsilon_{i j}=\frac{1}{2}\left(\frac{\partial u_{i}}{\partial x_{j}}+\frac{\partial u_{j}}{\partial x_{i}}\right)
$$

where $\sigma_{i j}$ is the effective stress in the tissue, $\varepsilon_{i j}$ is the strain tensor of the tumor tissue, $u_{i}$ are the displacement components, $\delta_{i j}$ is the Kronecker delta, $K$ is the drained bulk modulus of the tissue, $G$ is the shear modulus of the tissue, $p$ is the fluid pressure in the tissue, and $\eta$ is the volume of new tissue created per unit volume of tissue which is given by $\partial \eta / \partial t=S^{C}$, where $S^{C}$ is the rate of production of solid phase tumor tissue. ${ }^{4}$ Thus, if there is no displacement of the tissue (i.e., $\varepsilon_{i j}=0$ ), then the stress that builds up is proportional to the bulk modulus and the volume of material created per unit volume.

The constitutive equation (17) is supplemented by the two phase tissue mass conservation equations

$$
\frac{\partial \Phi^{C}}{\partial t}+\nabla \cdot\left(\mathbf{v}^{C} \Phi^{C}\right)=S^{C}, \quad \frac{\partial \Phi^{F}}{\partial t}+\nabla \cdot\left(\mathbf{v}^{F} \Phi^{F}\right)=S^{F},
$$

\footnotetext{
${ }^{4}$ This effective stress tensor can be derived by considering the solid phase and fluid phase stresses separately, $\sigma_{i j}^{s}=-\Phi^{s} p+2 G \varepsilon_{i j}+\left(K-\frac{2}{3} G\right) \varepsilon_{k k} \delta_{i j}-K \eta$ and $\sigma_{i j}^{f}=\Phi^{f} p \delta_{i j}$, thus the effective tissue stress $\sigma_{i j}^{s}+\sigma_{i j}^{f}$ will be given by (17) (see [109] for reference).
} 
where $\Phi^{C}$ and $\Phi^{F}$ are the tissue cell/matrix and fluid volume fractions, respectively, $\mathbf{v}^{C}$ is the cell/matrix velocity, $\mathbf{v}^{F}$ is the fluid velocity, and $S^{F}$ is the creation/degradation of the fluid phase. Conservation of matter in the tissue, $\Phi^{C}+\Phi^{F}=$ 1, implies that $\nabla \cdot\left(\mathbf{v}^{C} \Phi^{C}+\mathbf{v}^{F} \Phi^{F}\right)=S^{C}+S^{F}$. Using Darcy's flow for the fluid movement through the porous solid gives $\Phi^{F}\left(\mathbf{v}^{F}-\mathbf{v}^{C}\right)=-K_{H} \nabla p$, where $K_{H}$ is the hydraulic conductivity, while the assumption that the tumor may be described by two phases only implies that the new cell/matrix phase is formed from the fluid phase and vice versa, so that $S^{C}+S^{F}=0$. Finally the model is closed by a quasi-static force balance, $\nabla \cdot \underline{\underline{\sigma}}=\underline{0}$. The detailed biochemistry of tumor growth can be coupled into the model above through the growth term $S^{C}$, with equations added for nutrient diffusion.

We may combine the equations to find a single equation for the rate of solid phase dilatation $[93,110], e=\nabla \cdot \mathbf{v}^{C}$, as

$$
\frac{\partial e}{\partial t}=K_{H}\left(K+\frac{4}{3} G\right) \nabla^{2} e-K_{H} K \nabla^{2} S^{C} .
$$

Since the rate of dilatation of a spherically symmetric tumor is given by $e=\frac{1}{r^{2}} \frac{\partial}{\partial r}\left(r^{2} v_{r}^{C}\right)$, (19) completely describes the cell velocity inside a spherically symmetric tumor, and is the analogue of (9) for a compressible material. In particular, note that if we take the limit of incompressibility so that $K$ tends to infinity, then (19) reduces to (9). Note also that (19) needs to be solved both inside the tumor and in the gel, with different material constants in each and no production term in the gel. Across the tumor/agarose gel boundary there is a discontinuity of material properties, and continuity of stress and velocity is used to match the two regions together.

The model of Roose et al. [93] is very similar to earlier models of Jones et al. [107] and Chen, Byrne, and King [97], which treat the tumor as elastic rather than poroelastic. It is important to note that while biological tissue is often assumed to be incompressible when modeled as elastic, in a poroelastic model the Poisson ratio corresponds to that of the drained tissue, and may be significantly less than $\frac{1}{2}[111]$ (the effective incompressibility arising from the interstitial fluid). Roose et al. [93] measured $K$ directly in a confined compression experiment, and made quantitative predictions about the levels of stress and the volume of new tumor material created inside the multicell spheroids, estimating the level of stress to be about $10 \mathrm{mmHg}$.

Although the model (17)-(19) is much more complicated than (15), it is still based on linear poroelasticity, with the corresponding assumption of small displacements, while biological tissue and gels such as agarose undergo large deformations and exhibit nonlinear material properties. Note, though, that in a radially symmetric deformation the dilatation of the surrounding material is zero, so that there is no compaction of the gel. In this case its nonlinearity is likely to be less severe and the linear approximation may not be too bad.

In addition to the models mentioned above, there are several other models which consider the two phase nature of tumors $[52,63,94]$ and the hyperelastic nature of tumor tissue [95]. For example, a recent paper by Landman and Please [52] presents a two-phase (fluid and cell) model for tumor spheroid growth that aims to answer qualitative questions about the presence and size of the necrotic regions inside the tumor spheroid. The cell phase is considered to move in a Darcy-like fashion as in (5). It would be interesting to extend such models to see what effects the inclusion of cell traction forces $[112,113,114,115,116,117,118]$ might have on the model predictions. 
Another model presented by Byrne, King, and McElwain [96] considered a generic two-phase model for avascular tumor growth using the theory of mixtures. They assumed that the cell phase can be modeled as a viscous fluid, while the water phase can be modeled as an inviscid fluid. This paper provides a basis for further developments by considering specific tumor lines for which this type of model might be appropriate.

In concluding the continuum modeling section of this review, we point out the dual role of modeling. We focused initially on a specific model that was well-parameterized and which allowed detailed experimental predictions to be tested. However, in other situations we are not in a position to do such detailed modeling. In these cases, the role of modeling is in hypothesis testing and in helping to direct and formulate experiments. Such, more basic, conceptual models can also lead to experimental predictions, an example of which is the aforementioned discovery of an interstitial gap between advancing tumor cells and regressing normal cells [90].

3. Discrete Cell Population Models. With the huge advances in biotechnology, large amounts of data on phenomena occurring on a single cell scale are now available. This, combined with in vitro experiments using tumor spheroids, sandwich culture, etc., and high power confocal or multiphoton laser microscopy that enables tracking of individual cells in space and time, has brought about the possibility of modeling single-cell-scale phenomena and then using the techniques of upscaling to obtain information about the large-scale phenomena of tumor growth. There are several upscaling techniques; the most popular ones are cellular automata [31, 119, 120, 121, 122, 123, 124, 125, 126, 127, 128, 129, 130, 131, 132], lattice Boltzmann methods [9, 133], agent-based [129, 134], extended Potts [135], and the stochastic (Markov chain combined with Fokker-Planck equations) approach [97, 134, 136, 137, 138]. As in the case of phase-averaged continuum models discussed in the previous section, the main difficulty with the discrete cell-based models lies in their parameterization, and thus these models are more appropriate for giving qualitative insights, instead of detailed quantitative predictions.

In this section we will outline the main ideas behind the discrete approach for modeling cell populations in cancer. All of the main approaches (cellular automata, lattice Boltzmann, agent-based, extended Potts, etc.) consider the state of each cell or population of cells inside the tumor to be characterized by the vector variable

$$
\mathbf{w}=\{\mathbf{x}, \mathbf{v}, \mathbf{u}\},
$$

where $\mathbf{x}$ is the position of the cell, $\mathbf{v}$ is its velocity, and $\mathbf{u}$ is a vector characterizing the cell's internal biological state, which may incorporate its position in the cell cycle, its interaction with the local biochemical environment, etc. The mechanical interactions between the cells depend on their position $\mathbf{x}$ and velocity $\mathbf{v}$. This type of state vector has its origins in lattice Boltzmann and other statistical mechanics models, but we expect that the cell velocity plays a lesser role in tumor biology since "collision effects" between tumor cells are not comparable to those between gas molecules, for example. While it is in principle possible to write down a set of mechanical interactions between cells using statistical mechanics or Newton's laws of motion, this is not usually done since the resulting system is large and complicated and extremely computationally expensive, while at the same time many assumptions have to be made about the way cells interact so that its realism is not guaranteed. Thus it is more common to replace physical laws of cell motion such as force and mass balances with cell movement rules. Some general principles for these cell movement rules are described below. 
Since the cells of an automaton model correspond to biological cells (or collections of cells), it is easy and natural to incorporate the internal biochemical machinery of the biological cell into the internal state variables of the automaton cell. This is one of the main advantages of automaton models, and it makes it easy to consider heterogeneous populations of cells.

The difficulty with automaton models is realistically modeling cell motion. The first step in setting up the rules for cell motion is to partition the physical space into automaton cells. The simplest partition is to discretize into a regular lattice; rectangular lattices are usually chosen for simplicity. An alternative partition used by Kansal et al. [121] distributes the centers of automaton cells randomly and then uses a Voronoi tessellation to divide space up into cells.

The second modeling decision is whether the lattice is fixed in time or varies as the elements move. It is far simpler to consider a fixed lattice, with each automaton cell corresponding to either a biological cell or a vacant site, and with cells able to move into a nearby lattice site containing a vacant site. Of course, it is much more realistic to have lattice cells growing and moving, with the lattice evolving as the tumor grows, but such models are much harder to formulate and are therefore much rarer. In particular, while the rules of motion for fixed lattices can be formulated simply in terms of cells moving between lattice sites, if the lattice is free to move and the cells can grow, we are back into the territory of mass conservation and continuum mechanics to determine the rules for the motion of the cells.

To determine the rule for cell motion in a fixed lattice, most models assume that the interactions between an individual cell and the cells surrounding it are localized in space and occur only with cells inside a certain neighborhood $\mathrm{O}$, which is often the cell's nearest neighbors, but may extend further than this. A typical cellular automaton rule will say that if a location $\mathbf{x}$ is occupied by a cancer cell, this cell might proliferate with probability $k_{1}$, it might bind to an effector chemical, such as a growth factor or a factor causing the cell to die or become quiescent with probability $k_{2}$, or the cell might move to a neighboring site with probability $k_{3}$. The proliferation of cells depends on the levels of nutrients and waste products at any given point, and should ideally be derived from the biochemical pathways affecting the cell cycle. A relatively simple example would be an expression similar to (10) in section 2, where the levels of glucose, oxygen, and $\mathrm{pH}$ are considered to be influencing cell proliferation. In more sophisticated models cell proliferation may be the result of a network of ordinary differential equations modeling the internal cell machinery and signal transduction pathways. The probabilities $k_{1}, k_{2}$, and $k_{3}$ are usually taken to be independent of geometry and the mechanical environment of the cell, but these effects could in principle be incorporated also.

Once the decision has been taken to proliferate, the cell will divide into two daughter cells. On a deformable lattice this presents no problem, since the cell simply grows to be twice its normal size, and then splits into two, each of which is the same size as the original. However, on a fixed lattice this growth is taken to occur instantaneously, and the new cells immediately occupy two sites. One of these cells is usually assumed to occupy the original site of the dividing cell, but the other requires an extra space. There are several ways to determine where to put this new cell. Usually it is taken to move to a neighboring site, but we have to decide whether the surrounding cells should be pushed away to make space (and if so, to where should they be pushed?), or whether the cell can only move to a "vacant" site. Determining where to put the extra cell is one of the main problems with the fixed lattice approach. Because of the restrictions the fixed lattice imposes, it is extremely difficult to incorporate real- 


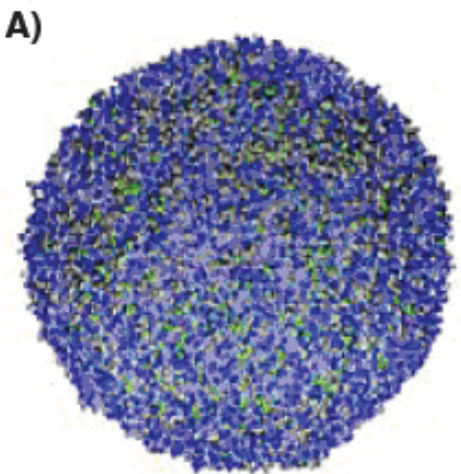

$200 \mu \mathrm{N} / \mathrm{m}$

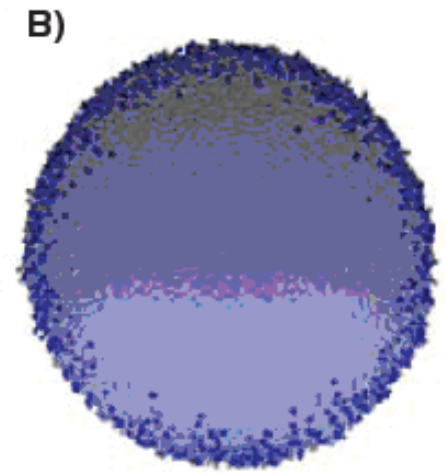

$600 \mu \mathrm{N} / \mathrm{m}$

Fig. 6 Simulation results with an agent-based model for two different cell anchorage strengths (reproduced from Galle, Loeffler, and Drasdo [139]). Green cells have contact with substrate and blue do not have contact with substrate.

istic physics or biology into such a movement rule. In addition, the particular lattice structure chosen may artificially influence the global behavior, in a manner similar to the grid effects which may be observed when numerically approximating solutions to partial differential equations. In the latter case it is possible to quantify this effect through mathematical analysis, but such a quantification is extremely difficult in cellular automata models. A similar dilemma is presented by a cell that does not proliferate but is highly motile. The movement of a cell to a neighboring site could depend on (a) the number of vacant neighboring sites, (b) the nutrient concentration gradient, (c) the levels of matrix molecules in neighboring sites, (d) the mechanical environment of the cell and cells in neighboring sites, and so on. Any number of these factors could be combined with a random motility assigned to each cell.

Different cellular automata models incorporate different levels of complexity into cell movement, birth, and death laws. Having described their general formulation we will now describe one or two specific examples of automaton models that have appeared in the literature. One of the first models considering discrete cell population migration using a complex cell cycle model, which is still considered to be sophisticated, was that of Duchting and Vogelsaenger [119], who conducted a series of three-dimensional simulations using the model in order to determine the effect of radiotherapy on tumors in a quantitative, well-parameterized manner (see the appendix for the description of their model). Another of the early models that appears to be reasonably well parameterized is that by Qi et al. [120], which is one of the least complicated models of its type and describes the minimal cellular automata rules that would reproduce the Gompertz law of cancer growth. ${ }^{5}$ See Figure 6 for a sample result from single-cell-based modeling reproduced from [139].

A recent three-dimensional cellular automaton model which does not use a regular lattice is that of Kansal et al. [121]. The model does not include nutrients or

\footnotetext{
${ }^{5}$ The Gompertz law states that the volume of the tumor $V(t)$ at time $t$ is given by $V(t)=$ $\left[\frac{V_{0}}{K}\right]^{\exp (-r t)} K$, where $V_{0}$ is the initial size of the tumor, $K$ is the equilibrium size, and $r$ is a positive constant.
} 
mechanical interaction between cells explicitly, but mimics the effects of both in a phenomenological way. The authors used a random fixed lattice and Voronoi tessellation, with the space that "belongs" to a single lattice site consisting of points that are nearer to this site than any other lattice site. Note though that the "cells" of the automaton each correspond to between $10^{3}$ and $10^{6}$ biological cells. A Delaunay triangulation is performed to connect the sites which share a common face, thus determining the neighbors of each of the lattice points. Having generated the lattice and determined the neighbors, Kansal et al. [121] ran a cell proliferation algorithm that determined the behavior of each lattice point at every time increment. In this model, the proliferation is determined by the distance of the cell from the tumor boundary to mimic the effects of nutrient diffusion and consumption; only cells within a certain distance from the boundary can proliferate. Similarly, cells a certain distance from the boundary become necrotic. An ad-hoc scaling of (tumor radius) $)^{2 / 3}$ is chosen for these distances. Mechanical pressure effects on cell proliferation are mimicked by reducing the probability of cell proliferation with the function $\left(1-r / R_{\max }\right)$, where $r$ is the radial position of the cell within the tumor and $R_{\max }$ is the maximum tumor radius. With these simple interaction rules, Kansal et al. [122] and [121] produced three-dimensional structures resembling tumors with different clonal tumor cell subpopulations.

Kansal et al. [122] and [121] included nutrient and cell mechanics effects into the model in a highly phenomenological manner, by simply setting the proliferation and death rates to be known functions of position. To make the model more biologically realistic, an obvious first extension would be to include the effects of nutrients and other chemicals explicitly in the model. One could start by using continuum equations for nutrient concentration and link this up to cellular automata models of the cell cycle and cell migration. Such an extension would lead to a hybrid model combining phenomena at the single cell level with continuum equations for macromolecular transport and comprising coupled discrete and continuum equations. A similar approach has been considered by Ferreira and coworkers [140, 141], who used a two-dimensional hybrid cellular automaton to model a population of cancer and normal cells. Growth factor concentrations are calculated from a continuous model with the cells acting as delta function source/sink terms, while the average nutrient levels (where averaging is performed over the domain of a cell and its von Neumann neighborhood of 5 cells in total) influence the probability of cell proliferation.

Whereas in most cellular automata models each lattice point consists of one or more biological cells, in the Potts model approach [135] each biological cell is made up of several lattice points and the movement of each individual cell is determined by some form of energy minimization. This formulation can take into account cell membrane tension, cell-cell and cell-matrix adhesion, chemotaxis, etc. Such an approach is more computationally intensive than automaton models and is suitable for the detailed modeling of small populations of cells.

A purely theoretical framework for dealing with the interaction of the immune system with discrete tumor cell populations using a lattice Boltzmann approach is presented in a recent review by Bellomo, Bellouquid, and De Angelis [133]. This approach utilizes the single-molecule-scale computational machinery known from fluid and gas dynamics to consider collective phenomena of tumor cells. Care must be taken when applying this type of statistical physics approach to cancer cells as they are much less mobile physically, but their behavior is significantly altered by changes in the intracellular and intercellular biochemical signaling networks. However, the benefit of lattice Boltzmann models, Markov chain, Fokker-Planck, etc., approaches originating 
from statistical mechanics is in the fact that there is already an existing framework from physics and fluid dynamics that deals with formalizing particle-particle or, in the case of cancer, cell-cell interactions. Using this theoretical framework, one can also recover the mean field continuum cell population models from the cellular automatatype particle interaction rules.

4. Discussion. While there have been some notable success stories of the application of mathematical models to cancer biology (a few of which have been discussed above), mathematics has much more to offer and there is still a long way to go. Since the biology and biochemistry of avascular tumor growth is highly complex and not completely understood, models have so far tended to be relatively simple, focusing on the interaction of certain generic processes. This is a necessary step as we extend our intuition and understanding of the novel types of interaction thrown up when modeling in the life sciences.

We see the role of mathematical modeling in cancer biology as twofold. On the one hand, mathematical models are able to verify (or dispute) hypothetical word models suggested by experimentalists. In this case the conclusion that a model is wrong can actually be more helpful, because it shows that the biologists should be looking for something else. Models can also help our intuition, provide a framework for thinking about a problem, and make predictions. If a model is well-parameterized, then these predictions can be quantitative, but even qualitative predictions can be significant. Gatenby and Gawlinski's work [90] is an example in which a simple threeequation model was able to explain a phenomenon that was previously thought to be influenced by many factors, with qualitative predictions that were largely independent of the specific parameter values.

In this respect, even purely theoretical studies of mathematical models have value, not just to mathematics (such as new mathematical challenges and the development of new techniques), but also to biology (such as providing generic insight into how different mechanisms may interact, or whether different modeling assumptions are likely to lead to qualitatively different behavior).

Having said this, we feel that the field of cancer biology is now reaching a stage of maturity at which the next step in the modeling process must be the careful parameterization of some of the models so that specific experimental predictions can be made and tested in close collaboration between experimentalists and theoreticians. As more and more biological details become available, particularly on the subcellular level as signal transduction pathways and biochemical networks are uncovered, these models are likely to become more and more complex. However, we need to guard against building overly complicated models which are poorly parameterized. While such models appear realistic, they may be little more than curve fitting, and may not give as much insight as simpler better-parameterized models.

In the future, large multiscale models are likely to be needed, as researchers attempt to integrate detailed subcellular information to make predictions on the tumor scale. It is not clear at present whether these models will be continuum or discrete, or a hybrid of the two, since there are problems associated with each approach.

Continuum models have the advantage of being more amenable to mathematical analysis and understanding, and are thus better able to aid intuition and give insight into underlying physical and biological principles. In addition they are likely to contain fewer parameters and can build on existing bodies of knowledge on continuum mechanics in other fields. On the other hand, the validity of the averaging over cells implicit in writing down a continuum model is questionable, especially when cells of 
more than one type are considered (in the final stages of development an avascular tumor spheroid is about 50 cells in diameter). It is also difficult to build the active nature of cells into mechanical laws for the tissue.

Discrete models have the advantage that they are perfectly adapted to modeling internal signaling networks within each cell (see the typical result of a discrete model in Figure 6 reproduced from [139]). There is in addition no requirement that all cells be the same; indeed, each can behave differently with no extra complication. The main problem with discrete models is that it is very difficult to build in realistic movement and growth laws based on biology and physics. In addition, they usually contain many more parameters and can only be analyzed computationally. Thus, while they can aid intuition through numerical experiments, they do not give as much insight.

In purely discrete models it is also difficult to build in interactions with diffusible chemicals. These are much easier to treat as a continuum, which has led to an emerging field of hybrid models in which individual cells are treated discretely but interact with other continuum fields. Such hybrid models are likely to develop further. An extension of this type of modeling which is likely to be significant in the future is multiscale models in which cells are treated both discretely and as a continuum, with key pieces of information passed between the two scales.

One area where mathematics can be of assistance is in providing a bridge between discrete and continuum models through homogenization. This can be either what we might call static, in which a discrete model is homogenized to give its continuum counterpart, or what we might call dynamic, in which key pieces of information from the discrete model are needed at the continuum level, and vice versa, so that the two models are coupled.

Finally, we are beginning to observe closer collaboration between mathematicians and experimentalists so that the pathway from model hypothesis and parameterization to testing of model predictions should become more rigorous in future. It is clear that while the large number of parameters needed to build the models cannot be measured directly for every single specific experimental condition, much progress could be made using standard techniques from physics and chemistry. For example, the motilities of biomolecules could be estimated using either the molecular weight of the molecule or its hydrodynamic radius and estimating the diffusion coefficient using the StokesEinstein relation. However, this type of parameter estimation is rarely done in the papers that are in the mathematics literature, and thus some models appear to be less useful to experimentalists than they actually could be.

While we have stressed the need for interdisciplinary collaboration between theoreticians and experimentalists, we also point out the need for interdisciplinary collaboration between theoreticians. For example, proper validation of the above models will require input from statisticians. The models above are all deterministic and ignore stochastic effects. It is important to study how such effects could change the behavior of the models. Modeling is part of the arsenal of tools biologists have at their disposal. As such, it must be used appropriately to answer the questions that it is good at answering (focused scientific questions which aim to address a specific biological aspect), and in conjunction with other biological tools that can address issues that modeling cannot.

\section{Appendix.}

Cell Cycle Rules. In this section we describe the model due to Duchting and Vogelsaenger [119] (see Figure 7). Each cell can stay in each stage for a given amount 


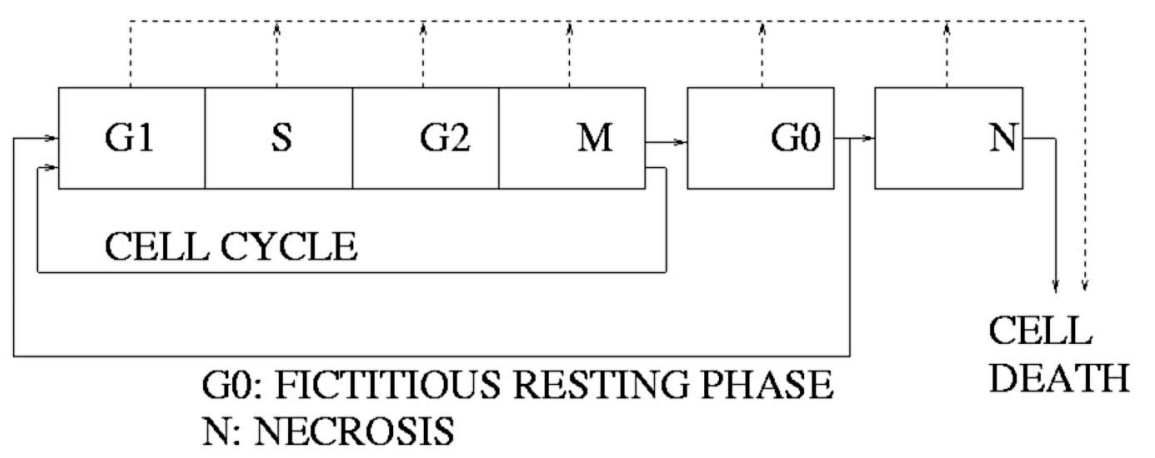

Fig. 7 Cellular automata rules from [119].

of time before moving to the next stage. The time it sits in each stage is drawn from a different normal distribution for different cell cycle phases. The difference between normal and tumor cells is that normal cells can go around the cell cycle only a finite number of times before arresting in a stable state, while tumor cells can go around the cell cycle indefinitely.

Cell Movement Rules. Normal cell movement rules: before entering mitosis, a cell checks if there is a free position nearby; if so, then division takes place and the new normal cell occupies the empty space. If there is no free space, then the normal cell will move to the resting phase.

Cancer cell movement rules: tumor cells can divide even when there is no free space around. Uniform random variables determine the direction where the new cancer cell will be created. All other cells will be shifted accordingly.

Acknowledgments. TR would like thank Dr. David C. Smith for help with the preparation of the manuscript. TR, SJC, and PKM would also like to thank three anonymous referees for their constructive criticism of the manuscript.

\section{REFERENCES}

[1] R.A. Gatenby and P.K. Maini, Mathematical oncology: Cancer summed up, Nature, 421 (2003), p. 321.

[2] F. Michor, Y. Iwasa, And M.A. Nowak, Dynamics of cancer progression, Nature Rev. Cancer, 4 (2004), pp. 197-205.

[3] R.M. Sutherland, Cell and environment interactions in tumor microregions: The multicell spheroid model, Science, 240 (1988), pp. 177-184.

[4] T. Udagawa, A. Fernandez, E.-G. Achilles, J. Folkman, and R.J. D'Amato, Persistence of microscopic human cancers in mice: Alterations in the angiogenic balance accompanies loss of tumor dormancy, Faseb J., 16 (2002), pp. 1361-1370.

[5] P. Yu, M. Mustata, L. Peng, J. J. Turek, M. R. Melloch, P. M. W. French, and D. D. NolTe, Holographic optical coherence imaging of rat osteogenic sarcoma tumor spheroids, 43 (2004), pp. 4862-4873.

[6] P. Carmeliet and R.K. Jain, Angiogenesis in cancer and other diseases, Nature, 407 (2000), pp. 249-257.

[7] M.V. Blagosklonny, Antiangiogenic therapy and tumor progression, Cancer Cell, 5 (2004), pp. 13-17.

[8] N. Mantzaris, S. Webb, and H.G. Othmer, Mathematical modeling of tumor-induced angiogenesis, J. Math. Biol., 95 (2004), pp. 111-187. 
[9] N. Bellomo And L. Preziosi, Modelling and mathematical problems related to tumor evolution and its interaction with the immune system, Math. Comput. Modelling, 32 (2000), pp. 413-452.

[10] S.A. Frank, Somatic mosaicism and cancer: Inference based on a conditional Luria-Delbruck distribution, J. Theoret. Biol., 223 (2003), pp. 405-412.

[11] R.A. Gatenby And B.R. Frieden, Application of information theory and extreme physical information to carcinogenesis, Cancer Res., 62 (2002), pp. 3675-3684.

[12] H. HASCHKE, A biokinetic model to describe consequences of inhibition/stimulation in DNAproofreading and repair-1. Development of the model, J. Theoret. Biol., 212 (2001), pp. $425-456$.

[13] Y. Iwasa, F. Michor, AND M.A. NOwAK, Evolutionary dynamics of invasion and escape, J. Theoret. Biol., 226 (2004), pp. 205-214.

[14] A. KAMB, Mutation load, functional overlap, and synthetic lethality in the evolution and treatment of cancer, J. Theoret. Biol., 223 (2003), pp. 205-213.

[15] T.V. Karpinets and B.D. Foy, Model of the developing tumorigenic phenotype in mammalian cells and the roles of sustained stress and replicative senescence, J. Theoret. Biol., 227 (2004), pp. 253-264.

[16] N.L. Komarova, A. Sengupta, And M.A. Nowak, Mutation-selection networks of cancer initiation: Tumor suppressor genes and chromosomal instability, J. Theoret. Biol., 223 (2003), pp. 433-450.

[17] F. Michor, S.A. Frank, R.M. May, Y. Iwasa, and M.A. Nowak, Somatic selection for and against cancer, J. Theoret. Biol., 225 (2003), pp. 377-382.

[18] J.B. Plotkin And M.A. NowAK, The different effects of apoptosis and DNA repair on tumorigenesis, J. Theoret. Biol., 214 (2002), pp. 453-467.

[19] R.V. Sole And T.S. Deisboeck, An error catastrophe in cancer?, J. Theoret. Biol., 228 (2004), pp. 47-54.

[20] R.K. JaIn, Delivery of molecular and cellular medicine to solid tumors, Advances in Drug Delivery Reviews, 46 (2001), pp. 149-168.

[21] R.P. Araujo And D.L.S. McElwain, A history of the study of solid tumor growth: The contribution of mathematical modelling, Bull. Math. Biol., 66 (2004), pp. 1039-1091.

[22] J.A. ADAM, Mathematical models of perivascular spheroid development and catastrophetheoretic description of rapid metastatic growth/tumor remission, Invastion and Metastasis, 16 (1996), pp. 247-267.

[23] H.M. ByRne, The role of mathematics in solid tumour growth, Math. Today, 35 (1999), pp. $59-89$.

[24] J.J. Casciari, S.V. Sotirchos, and R.M. Sutherland, Mathematical modelling of microenvironment and growth in EMT6/Ro multicellular tumour spheroids, Cell Proliferation, 25 (1992), pp. 1-22.

[25] A.C. BuRTon, Rate of growth of solid tumours as a problem of diffusion, Growth, 30 (1966), pp. $157-176$.

[26] J.A. AdAm, A simplified mathematical model of tumor growth, Math. Biosci., 81 (1986), pp. 229-244.

[27] J.A. ADAM, A mathematical model of tumor growth. II. Effects of geometry and spatial uniformity on stability, Math. Biosci., 86 (1987), pp. 183-211.

[28] J.A. Adam, A mathematical model of tumor growth. III. Comparison with experiments, Math. Biosci., 86 (1987), pp. 213-227.

[29] J.A. Adam and S.A. Maggelakis, Mathematical models of tumor growth. IV. Effects of a necrotic core, Math. Biosci., 97 (1989), pp. 121-136.

[30] J.A. Adam And S.A. Maggelakis, Diffusion regulated characteristics of a spherical perivascular carcinoma, Bull. Math. Biol., 52 (1990), pp. 549-582.

[31] A.R.A. Anderson, M.A.J. Chaplain, E.L. Newman, R.J.C. Steele, and A.M. ThompSON, Mathematical modelling of tumour invasion and metastasis, J. Theoret. Medicine, 2 (2000), pp. 129-154.

[32] B.H. ARVE AND A.I. LIAPIS, Oxygen tension in tumors predicted by diffusion with absorption model involving a moving free boundary, Math. Comput. Modelling, 10 (1988), pp. 159174 .

[33] H.M. Byrne and M.A.J. Chaplain, Growth of nonnecrotic tumours in the presence and absence of inhibitors, Math. Biosci., 130 (1995), pp. 151-181.

[34] H.M. Byrne And M.A.J. Chaplain, Growth of necrotic tumours in the presence and absence of inhibitors, Math. Biosci., 135 (1996), pp. 187-216.

[35] H.M. Byrne AND S.A. Gourley, The role of growth factors in avascular tumour growth, Math. Comput. Modelling, 26 (1997), pp. 35-55. 
[36] H.M. BYRne, The importance of intercellular adhesion in the development of carcinomas, IMA J. Math. Appl. Medicine Biol., 14 (1997), pp. 305-323.

[37] H.M. Byrne And M.A.J. Chaplain, Free boundary value problems associated with the growth and development of multicellular spheroids, European J. Appl. Math., 8 (1997), pp. 639658.

[38] H.M. Byrne and M.A.J. Chaplain, Necrosis and apoptosis: Distinct cell loss mechanisms in a mathematical model of avascular tumour growth, J. Theoret. Medicine, 1 (1998), pp. 223-235.

[39] H.M. ByRne, A weakly nonlinear analysis of a model of avascular solid tumour growth, J. Math. Biol., 39 (1999), pp. 59-89.

[40] H.M. Byrne And P. MatThews, Asymmetric growth of models of avascular solid tumours: Exploiting symmetries, IMA J. Math. Appl. Medicine Biol., 19 (2002), pp. 1-29.

[41] M.A.J. Chaplain And N.F. BRITton, On the concentration profile of a growth inhibitory factor in multicell spheroids, Math. Biosci., 115 (1993), pp. 233-245.

[42] M.A.J. Chaplain, D.L. Benson, and P.K. Maini, Nonlinear diffusion of a growth inhibitory factor in multicell spheroids, Math. Biosci., 121 (1994), pp. 1-13.

[43] V. Cristini, J. Lowengrub, And Q. Nie, Nonlinear simulation of tumor growth, J. Math. Biol., 46 (2003), pp. 191-224.

[44] A.S. Deakin, Model for the growth of a solid in vitro tumor, Growth, 39 (1975), pp. 159-165.

[45] S.B. Cui AND A. Friedman, A free boundary problem for a singular system of differential equations: An application to a model of tumor growth, Trans. Amer. Math. Soc., 355 (2003), pp. 3537-3590.

[46] F.L. Degner and R.M. Sutherland, Mathematical modelling of oxygen supply and oxygenation in tumor tissues: Prognostic, therapeutic and experimental implications, Internat. J. Radiation Oncology Biol. Phys., 15 (1988), pp. 391-397.

[47] S.J. FRANKS AND J.R. KING, Interactions between a uniformly proliferating tumour and its surroundings: Uniform material properties, Math. Medicine Biol., 20 (2003), pp. 47-89.

[48] J.P. Freyer AND R.M. Sutherland, Regulation of growth saturation and development of necrosis in EMT6/Ro multicellular spheroids by glucose and oxygen supply, Cancer Res., 46 (1986), pp. 3504-3512.

[49] H.P. Greenspan, Models for the growth of a solid tumor by diffusion, Stud. Appl. Math., 52 (1972), pp. 317-340.

[50] H.P. GreEnspan, On the growth and stability of cell cultures and solid tumors, J. Theoret. Biol., 56 (1975), pp. 229-242.

[51] U. Grossmann, Profiles of oxygen partial pressure and oxygen consumption inside multicellular spheroids, Recent Results in Cancer Res., 95 (1984), pp. 150-159.

[52] K.A. Landman and C.P. Please, Tumour dynamics and necrosis: Surface tension and stability, IMA J. Math. Appl. Medicine Biol., 18 (2001), pp. 131-158.

[53] J. Landry, J.P. Freyer, And R.M. Sutherland, A model for the growth of multicellular spheroids, Cell and Tissue Kinetics, 15 (1982), pp. 585-594.

[54] J. LANDRY AND J.P. FREYER, Regulatory mechanisms in spheroid aggregates of normal and cancerous cells, Recent Results in Cancer Res., 95 (1984), pp. 50-66.

[55] B.P. Marchant, J. Norbury, and A.J. Perumpanani, Traveling shock waves arising in a model of malignant invasion, SIAM J. Appl. Math., 60 (2000), pp. 463-476.

[56] B.P. Marchant, J. Norbury, And J.A. Sherratt, Travelling wave solutions to a haptotaxisdominated model of malignant invasion, Nonlinearity, 14 (2001), pp. 1653-1671.

[57] M. Marusic, Z. Bajzer, J.P. Freyer, And S. Vuk-Pavlovic, Modeling autostimulation of growth in multicellular tumor spheroids, Internat. J. Biomedical Comput., 29 (1991), pp. $149-158$

[58] D.L.S. McElwain And P.J. Ponzo, A model for the growth of a solid tumor with non-uniform oxygen consumption, Math. Biosci., 35 (1977), pp. 267-279.

[59] D.L.S. McElwain, A reexamination of oxygen diffusion in a spheroid cell with MichaelisMenten oxygen uptake kinetics, J. Theoret. Biol., 71 (1978), pp. 255-267.

[60] D.L.S. McElwain And G.J. Pettet, Cell migration in multicell spheroids: Swimming against the tide, Bull. Math. Biol., 55 (1993), pp. 655-674.

[61] W. Mueller-Klieser, Method for the determination of oxygen consumption rates and diffusion coefficients in multicellular spheroids, Biophys. J., 46 (1984), pp. 343-348.

[62] G.J. Pettet, C.P. Please, M.J. Tindall, and D.L. McElwain, The migration of cells in multicell tumor spheroids, Bull. Math. Biol., 63 (2001), pp. 231-257.

[63] C.P. Please, G.J. Pettet, and D.L.S. McElwain, A new approach to modelling the formation of necrotic regions in tumours, Appl. Math. Lett., 11 (1998), pp. 89-94.

[64] C.P. Please, G.J. Pettet, And D.L.S. McElwain, Avascular tumour dynamics and necrosis, Math. Models Methods Appl. Sci., 9 (1999), pp. 569-579. 
[65] J.A. SHERRATt, Traveling wave solutions of a mathematical model for tumor encapsulation, SIAM J. Appl. Math., 60 (1999), pp. 392-407.

[66] R.M. Shymko And L. Glass, Cellular and geometric control of tissue growth and mitotic instability, J. Theoret. Biol., 63 (1976), pp. 355-374.

[67] J.P. WARD AND J.R. KING, Mathematical modelling of avascular tumour growth, IMA J. Math. Appl. Medicine Biol., 14 (1997), pp. 36-69.

[68] J.P. WARD AND J.R. KING, Mathematical modelling of avascular tumour growth. II. Modelling growth saturation, IMA J. Math. Appl. Medicine Biol., 16 (1999), pp. 171-211.

[69] J.P. WARD AND J.R. KInG, Mathematical modelling of the effects of mitotic inhibitors on avascular tumour growth, J. Theoret. Medicine, 1 (1999), pp. 287-311.

[70] S.A. Maggelakis And J.A. Adam, Mathematical model of perivascular growth of a spherical carcinoma, Math. Comput. Modelling, 13 (1990), pp. 23-38.

[71] J.A. Sherratt, Cellular growth control and travelling waves of cancer, SIAM J. Appl. Math., 53 (1993), pp. 1713-1730.

[72] D.L.S. McElwain AND L.E. Morris, Apoptosis as a volume loss mechanism in mathematical models of solid tumor growth, Math. Biosci., 39 (1978), pp. 147-157.

[73] L.M. Sander and T.S. Deisboeck, Growth patterns of microscopic brain tumors, Phys. Rev. E, 66 (2002), pp. 66-73.

[74] M.A.J. Chaplain, Avascular growth, angiogenesis and vascular growth in solid tumours: The mathematical modelling of the stages of tumour development, Math. Comput. Modelling, 23 (1996), pp. 47-87.

[75] J.A. Sherratt and M.A.J. Chaplain, A new mathematical model for avascular tumour growth, J. Math. Biol., 43 (2001), pp. 291-312.

[76] K.E. Thompson And H.M. Byrne, Modelling the internalisation of labelled cells in tumour spheroids, Bull. Math. Biol., 61 (1999), pp. 601-623.

[77] S. Tohya, A. Mochizuki, S. Imayama, and Y. Iwasa, On rugged shape of skin tumor (basal cell carcinoma), J. Theoret. Biol., 194 (1998), pp. 65-78.

[78] S. Habib, C. Molina-Paris, and T.S. Deisboeck, Complex dynamics of tumors: Modeling an emerging brain tumor system with coupled reaction-diffusion equations, Phys. A, 327 (2003), pp. 501-524.

[79] J.P. WARD AND J.R. KING, Mathematical modelling of drug transport in tumour multicell spheroids and monolayer cultures, Math. Biosci., 181 (2003), pp. 177-207.

[80] M.A.J. Chaplain, M. Ganesh, and I.G. Graham, Spatio-temporal pattern formation on spherical surfaces: Numerical simulation and application to solid tumor growth, J. Math. Biol., 42 (2001), pp. 387-423.

[81] K. MASEIDE AND E. Rofstad, Mathematical modeling of chronical hypoxia in tumors considering potential doubling time and hypoxic cell lifetime, Radiotherapy and Oncology, 54 (2000), pp. 171-177.

[82] L.M. Wein, J.T. Wu, A.G. Innculescu, And R.K. Puri, A mathematical model of the impact of infused targeted cytotoxic agents on brain tumours: Implications for detection, design and delivery, Cell Proliferation, 35 (2002), pp. 343-361.

[83] J.J. Casciari, The Effects of the Diffusion and Reaction of Nutrients and Metabolic Waste Products on the Growth and Microenvironment of Multicellular Tumor Spheroids, Ph.D. thesis, Department of Chemical Engineering, University of Rochester, Rochester, NY, 1989 .

[84] R.M. Youngson, Collins Dictionary of Medicine, Harper Collins, UK, 1992.

[85] J.J. Casciari, S.V. Sotirchos, And R.M. Sutherland, Nutrient concentration and $p H$ gradients in multicellular tumor spheroids and their effects on growth and metabolism, Paper 192c, AICHE Annual Meeting, Chicago, 1990.

[86] F.J. BuRkowski, A computer simulation of the growth of a tumor in vitro, Computer Programs in Biomedicine, 7 (1977), pp. 203-210.

[87] H.M. Byrne, The effect of time delays on the dynamics of avascular tumour growth, Math. Biosci., 144 (1997), pp. 83-117.

[88] M.J. Dorie, R.F. Kallman, D.F. Rapacchietta, D. Van Antwerp, and Y.R Huang, Migration and internalisation of cells and polysterene microspheres in tumor cell spheroids, Experimental Cell Res., 141 (1982), pp. 201-209.

[89] M.J. Dorie, R.F. Kallman, And M.A. CoYne, Effect of cytochalasin b, nocodazole and irradiation on migration and internalization of cells and microspheres in tumor spheroids, Experimental Cell Res., 166 (1986), pp. 370-378.

[90] R.A. Gatenby and E.T. Gawlinski, A reaction-diffusion model of cancer invasion, Cancer Res., 56 (1996), pp. 5745-5753.

[91] R.A. Gatenby, E.T. Gawlinski, A.F. Gmitro, B. Kaylor, and R.J. Gillies, Acid-mediated tumor invasion: A multidisciplinary study, 66 (2006), pp. 5216-5223. 
[92] H.M. Byrne and M.A.J. Chaplain, Modelling the role of cell-cell adhesion in the growth and development of carcinomas, Math. Comput. Modelling, 24 (1996), pp. 1-17.

[93] T. Roose, P.A. Netti, L.L. Munn, Y. Boucher, and R.K. Jain, Solid stress generated by spheroid growth estimated using a linear poroelasticity model, Microvascular Res., 66 (2003), pp. 204-212.

[94] C.J.W. Breward, H.M. Byrne, and C.E. Lewis, The role of cell-cell interaction in a two phase model for avascular tumour growth, J. Math. Biol., 45 (2002), pp. 125-152.

[95] D. Ambrosi And F. Mollica, The role of stress in the growth of a multicell spheroid, J. Math. Biol., 48 (2003), pp. 477-499.

[96] H.M. Byrne, J.R. King, AND D.L.S. McElwain, A two-phase model of solid tumor growth, Appl. Math. Lett., 16 (2003), pp. 567-573.

[97] C.Y. Chen, H.M. Byrne, And J.R. KIng, The influence of growth-induced stress from the surrounding medium on the development of multicell spheroids, J. Math. Biol., 43 (2001), pp. 191-220.

[98] H.M. Byrne And L. Preziosi, Modelling solid tumour growth using the theory of mixtures, Math. Medicine Biol., 20 (2003), pp. 341-366.

[99] B.D. MacArthur And C.P. Please, Residual stress generation and necrosis formation in multi-cell tumour spheroids, J. Math. Biol., 49 (2004), pp. 537-552.

[100] S.R. Lubkin AND T.L. JACKSOn, Multiphase mechanics of capsule formation in tumors, J. Biomech. Engrg., 124 (2002), pp. 1-7.

[101] R. Wasserman and R. Acharya, A patient-specific in vivo tumour model, Math. Biosci., 136 (1996), pp. 111-140.

[102] T.L. Jackson And H.M. Byrne, A mechanical model of tumor encapsulation and transcapsular spread, Math. Biosci., 180 (2002), pp. 307-328.

[103] M.A.J. Chaplain And B.D. Sleeman, Modelling the growth of solid tumors and incorporating a method for their classification using nonlinear elasticity theory, J. Math. Biol., 31 (1993), pp. 431-473.

[104] P. Canadas, V.M. Laurent, C. Oddou, D. Isabey, and S. Wendling, A cellular tensegrity model to analyse the structural viscoelasticity of the cytoskeleton, J. Theoret. Biol., 218 (2002), pp. 155-173.

[105] G. Helmlinger, P.A. Netti, H.C. Lichtenbeld, R.J. Melder, And R.K. Jain, Solid stress inhibits the growth of multicellular tumor spheroids, Nature Biotechnology, 15 (1997), pp. 778-783.

[106] P.A. Netti, D.A. Berk, M.A. Swartz, A.J. Grodzinsky, and R.K. Jain, Role of extracellular matrix assembly in interstitial transport in solid tumors, Cancer Res., 60 (2000), pp. 2497-2503.

[107] A.F. Jones, H.M. Byrne, J.S. Gibson, And J.W. Dold, A mathematical model of the stress induced during avascular tumor growth, J. Math. Biol., 40 (2000), pp. 473-499.

[108] L.D. Landau and E.M. Lifshitz, Theory of Elasticity, 3rd ed., Reed Educational and Professional, Oxford, UK, 1986.

[109] V.C. Mow, M.K. Kwan, W.M. Lai, and M.H. Holmes, A finite deformation theory for nonlinearly permeable soft hydrated biological tissues, in Frontiers in Biomechanics, S. Schmid-Schonbein, L.Y. Woo, and B.W. Zweifach, eds., Springer-Verlag, New York, 1986, pp. $153-179$.

[110] P.A. Netti, L.T. Baxter, Y. Boucher, R. Skalak, And R.K. Jain, Macro- and microscopic fluid transport in living tissues: Application to solid tumors, AIChE J., 43 (1997), pp. 818-834.

[111] S. Vogel, Comparative Biomechanics. Life's Physical World, Princeton University Press, Princeton, NJ, 2003.

[112] Y. Boucher, R.K. Jain, And K. Burton, Traction forces and rates of migration in several cancer cell lines, Molecular Biology of the Cell, 11 (2000), p. 439.

[113] K. Burton, R.K. Jain, And Y. Boucher, Traction forces and migration in cancer, endothelial, and fibroblast cells, Biophys. J., 80 (2001), p. 1439.

[114] K. Burton, J. PARK, And D.L. TAYLOR, Lamellar traction forces are centripetal and oriented in a continuous and symmetrical distribution about the direction of movement in crawling cells, Molecular Biology of the Cell, 7 (1996), pp. 2270-2270.

[115] K. Burton, J. PARK, And D.L. TAYlor, The leading lamellum of crawling cells generates traction forces in a radial array to pull cells forward, Biophys. J., 72 (1997), p. A187.

[116] K. Burton, J.H. Park, And D.L. TAYlor, Keratocytes generate traction forces in two phases, Molecular Biology of the Cell, 10 (1999), pp. 3745-3769.

[117] K. Burton AND D.L. TAYlor, Traction forces of cytokinesis and cell motility, Molecular Biology of the Cell, 8 (1997), pp. 975-975. 
[118] K. Burton And D.L. TAYLOR, Traction forces of cytokinesis measured with optically modified elastic substrata, Nature, 385 (1997), pp. 450-454.

[119] W. Duchting And T. Vogelsaenger, Recent progress in modelling and simulation of threedimensional tumor growth and treatment, Biosystems, 18 (1985), pp. 493-412.

[120] A.-S. QI, X. Zheng, C.Y. Du, And B.S. An, A cellular automaton model of cancerous growth, J. Theoret. Biol., 161 (1993), pp. 1-12.

[121] A.R. Kansal, S. Torquato, G.R. Harsh IV, E.A. Chiocca, and T.S. Deisboeck, Simulated brain tumor growth dynamics using a three-dimensional cellular automaton, J. Theoret. Biol., 203 (2000), pp. 367-382.

[122] A.R. Kansal, S. Torquato, E.A. Chiocca, and T.S. Deisboeck, Emergence of a subpopulation in a computational model of tumor growth, J. Theoret. Biol., 207 (2000), pp. 431-441.

[123] E.L. Stott, N. Britton, J.A. Glazier, And M. Zajac, Stochastic simulation of benign avascular tumour growth using the Potts model, Math. Comput. Modelling, 30 (1999), pp. $183-198$.

[124] J. Smolle AND H. Stettner, Computer simulation of tumour cell invasion by a stochastic growth model, J. Theoret. Biol., 160 (1993), pp. 63-72.

[125] J. Smolle, Cellular automation simulation of tumour growth-equivocal relationships between simulation parameters and morphologic pattern features, Analytical Cell Pathology, 17 (1998), pp. 71-82.

[126] P.P. Delsanto, A. Romano, M. Scalerandi, and G.P. Pescarmona, Analysis of a "phase transition" from tumor growth to latency, Phys. Rev. E, 62 (2000), pp. 2547-2554.

[127] D. Drasdo, R. Kree, and J.S. McCaskill, Monte-Carlo approach to tissue cell populations, Phys. Rev. E, 52 (1995), pp. 6635-6657.

[128] T. Alarcon, H.M. Byrne, And P.K. Maini, A cellular automaton model for tumour growth in inhomogeneous environment, J. Theoret. Biol., 225 (2003), pp. 257-274.

[129] D. Drasdo and S. Hohme, Individual-based approaches to birth and death in avascular tumors, Math. Comput. Modelling, 37 (2003), pp. 1163-1175.

[130] A. Deutsch And S. Dormann, Modelling of avascular tumour growth with a hybrid cellular automaton, In Silico Biology, 2 (2002), pp. 1-14.

[131] D. Drasdo, A Monte-Carlo approach to growing solid non-vascular tumors, in Dynamical Networks in Physics and Biology at the Frontier of Physics and Biology, D. Beysens and G. Forgacs, eds., Springer-Verlag, Berlin, Heidelberg, 1998, pp. 171-185.

[132] D. Drasdo, S. Dormann, S. Hoenme, and A. Deutsch, Cell-based models of avascular tumor growth, in Function and Regulation of Cellular Systems: Experiments and Models, A. Deutsch, J.H. Falcke, and W. Zimmermann, eds., Birkhäuser, Basel, 2004, pp. 367-378.

[133] N. Bellomo, A. Bellouquid, And E. De Angelis, The modelling of immune competition by generalised kinetic (Boltzmann) models: Review and research perspectives, Math. Comput. Modelling, 37 (2003), pp. 65-86.

[134] Y. Mansury, M. Kimura, J. Lobo, and T.S. Diesboeck, Emerging patterns in tumor systems: Simulating the dynamics of multicellular clusters with an agent-based spatial agglomeration model, J. Theoret. Biol., 219 (2002), pp. 343-370.

[135] S. Turner And J.A. Sherratt, Intercellular adhesion and cancer invasion: A discrete simulation using the extended Potts model, J. Theoret. Biol., 216 (2002), pp. 85-100.

[136] W.Y. Chen, P.R. Annamreddy, And L.T. Fan, Modeling growth of a heterogeneous tumor, J. Theoret. Biol., 221 (2003), pp. 205-227.

[137] R. Wette, I.N. Katz, And E.Y. Rodin, Stochastic processes for solid tumor kinetics I. Surface-regulated growth, Math. Biosci., 19 (1974), pp. 231-255.

[138] R. Wette, I.N. Katz, And E.Y. Rodin, Stochastic processes for solid tumor kinetics II. Diffusion-regulated growth, Math. Biosci., 21 (1974), pp. 311-338.

[139] J. Galle, M. Loeffler, AND D. Drasdo, Modeling the effect of deregulated proliferation and apoptosis on the growth dynamics of epithelial cell populations in vitro, Biophys. J., 88 (2005), pp. 62-75.

[140] S.C. Ferreira, Jr., M.L. Martins, And M.J. Vilela, A growth of primary cancer (II). New rules, progress curves and morphology transitions, Phys. A, 272 (1999), pp. 245-256.

[141] S.C. Ferreira, Jr., M.L. Martins, and M.J. Vilela, A growth model for primary cancer, Phys. A, 261 (1998), pp. 569-580.

[142] D.E. Ingber, Tensegrity I. Cell structure and hierarchical systems biology, J. Cell Sci., 116 (2003), pp. 1157-1173.

[143] S. HuAng And D.E. IngBer, The structural and mechanical complexity of cell-growth control, Nature Cell Biol., 1 (1999), pp. E131-E138. 